\title{
An overpartition analogue of the Andrews-Göllnitz-Gordon theorem
}

\author{
Thomas Y. He ${ }^{1}$, Kathy Q. Ji², Allison Y.F. Wang ${ }^{3}$ and Alice X.H. Zhao ${ }^{4}$ \\ ${ }^{1,2,3}$ Center for Applied Mathematics, Tianjin University, Tianjin 300072, P.R. China \\ ${ }^{4}$ College of Science, Tianjin University of Technology, Tianjin 300384, P.R. China \\ ${ }^{1}$ heyao@tju.edu.cn, ${ }^{2}$ kathyji@tju.edu.cn, ${ }^{3}$ wangyifang@tju.edu.cn, \\ ${ }^{4}$ zhaoxh@email.tjut.edu.cn
}

\begin{abstract}
In 1967, Andrews found a combinatorial generalization of the Göllnitz-Gordon theorem, which can be called the Andrews-Göllnitz-Gordon theorem. In 1980, Bressoud derived a multisum Rogers-Ramanujan-type identity, which can be considered as the generating function counterpart of the Andrews-Göllnitz-Gordon theorem. Lovejoy gave an overpartition analogue of the Andrews-Göllnitz-Gordon theorem for $i=k$. In this paper, we give an overpartition analogue of this theorem in the general case. By using Bailey's lemma and a change of base formula due to Bressoud, Ismail and Stanton, we obtain an overpartition analogue of Bressoud's identity. We then give a combinatorial interpretation of this identity by introducing the Göllnitz-Gordon marking of an overpartition, which yields an overpartition analogue of the Andrews-Göllnitz-Gordon theorem.
\end{abstract}

Keywords: The Göllnitz-Gordon theorem, Overpartition, Bailey pair, Göllnitz-Gordon marking

AMS Classifications: 05A17, 11P84.

\section{Introduction}

The purpose of this paper is to give an overpartition analogue of the Andrews-GöllnitzGordon theorem in the general case. In 1967, Andrews [3] found the following combinatorial generalization of the Göllnitz-Gordon identities [15, 17], which has been called the Andrews-Göllnitz-Gordon theorem.

Theorem 1.1 (Andrews-Göllnitz-Gordon). For $k \geq i \geq 1$, let $C_{k, i}(n)$ denote the number of partitions $\lambda$ of $n$ of the form $\left(1^{f_{1}}, 2^{f_{2}}, 3^{f_{3}}, \ldots\right.$ ), where $f_{t}(\lambda)$ (or $f_{t}$ for short) denotes the number of occurrences of $t$ in $\lambda$, such that

(1) $f_{1}(\lambda)+f_{2}(\lambda) \leq i-1$;

(2) $f_{2 t+1}(\lambda) \leq 1$; 
(3)

$f_{2 t}(\lambda)+f_{2 t+1}(\lambda)+f_{2 t+2}(\lambda) \leq k-1$

For $k \geq i \geq 1$, let $D_{k, i}(n)$ denote the number of partitions of $n$ into parts $\not \equiv 2(\bmod 4)$ and $\not \equiv 0, \pm(2 i-1)(\bmod 4 k)$.

Then for $k \geq i \geq 1$ and $n \geq 0$,

$$
C_{k, i}(n)=D_{k, i}(n)
$$

The Andrews-Göllnitz-Gordon theorem was motivated by a combinatorial generalization of the Rogers-Ramanujan identities due to Gordon [16].

Theorem 1.2 (Rogers-Ramanujan-Gordon). For $k \geq i \geq 1$, let $B_{k, i}(n)$ denote the number of partitions $\lambda$ of $n$ of the form $\left(1^{f_{1}}, 2^{f_{2}}, 3^{f_{3}}, \ldots\right)$ such that

(1) $f_{1}(\lambda) \leq i-1$

(2) $f_{t}(\lambda)+f_{t+1}(\lambda) \leq k-1$.

For $k \geq i \geq 1$, let $A_{k, i}(n)$ denote the number of partitions of $n$ into parts $\not \equiv 0, \pm i$ $(\bmod 2 k+1)$.

Then for $k \geq i \geq 1$ and $n \geq 0$,

$$
A_{k, i}(n)=B_{k, i}(n)
$$

The analytic proof of Theorem [1.2 was provided by Andrews [2], and in [4], he discovered the following identity, which has been called the Andrews-Gordon identity, see Kurşungöz [18].

Theorem 1.3 (Andrews). For $k \geq i \geq 1$,

$$
\sum_{N_{1} \geq N_{2} \geq \ldots \geq N_{k-1} \geq 0} \frac{q^{N_{1}^{2}+N_{2}^{2}+\cdots+N_{k-1}^{2}+N_{i}+\cdots+N_{k-1}}}{(q ; q)_{N_{1}-N_{2}}(q ; q)_{N_{2}-N_{3}} \cdots(q ; q)_{N_{k-1}}}=\frac{\left(q^{i}, q^{2 k+1-i}, q^{2 k+1} ; q^{2 k+1}\right)_{\infty}}{(q ; q)_{\infty}} .
$$

Here and in the sequel, we adopt the standard notation [5]:

$$
(a ; q)_{\infty}=\prod_{i=0}^{\infty}\left(1-a q^{i}\right), \quad(a ; q)_{n}=\frac{(a ; q)_{\infty}}{\left(a q^{n} ; q\right)_{\infty}}
$$

and

$$
\left(a_{1}, a_{2}, \ldots, a_{m} ; q\right)_{\infty}=\left(a_{1} ; q\right)_{\infty}\left(a_{2} ; q\right)_{\infty} \cdots\left(a_{m} ; q\right)_{\infty}
$$

Theorem 1.3 can be considered as the generating function version of Theorem 1.2. It is evident that the generating function of $A_{k, i}(n)$ in Theorem 1.2 equals the right-hand side of (1.1). By using $q$-difference equations, Andrews [4] showed that the generating function of $B_{k, i}(n)$ in Theorem 1.2 equals the left-hand side of (1.1). In particular, he obtained the following formula for the generating function of $B_{k, i}(m, n)$, where $B_{k, i}(m, n)$ denotes the number of partitions enumerated by $B_{k, i}(n)$ with exactly $m$ parts. 
Theorem 1.4 (Andrews). For $k \geq i \geq 1$,

$$
\sum_{m, n \geq 0} B_{k, i}(m, n) x^{m} q^{n}=\sum_{N_{1} \geq N_{2} \geq \ldots \geq N_{k-1} \geq 0} \frac{q^{N_{1}^{2}+N_{2}^{2}+\cdots+N_{k-1}^{2}+N_{i}+\cdots+N_{k-1}} x^{N_{1}+\cdots+N_{k-1}}}{(q ; q)_{N_{1}-N_{2}}(q ; q)_{N_{2}-N_{3}} \cdots(q ; q)_{N_{k-1}}} .
$$

Kurşungöz [18] gave a combinatorial proof of (1.2) by introducing the notion of the Gordon marking of a partition.

The generating function version of the Andrews-Göllnitz-Gordon theorem 1.1 was found by Bressoud [10, Eq. (3.8)] in 1980.

Theorem 1.5 (Bressoud). For $k \geq i \geq 1$,

$$
\begin{aligned}
& \sum_{N_{1} \geq N_{2} \geq \ldots \geq N_{k-1} \geq 0} \frac{\left(-q^{1-2 N_{1}} ; q^{2}\right)_{N_{1}} q^{2\left(N_{1}^{2}+\cdots+N_{k-1}^{2}+N_{i}+\cdots+N_{k-1}\right)}}{\left(q^{2} ; q^{2}\right)_{N_{1}-N_{2}}\left(q^{2} ; q^{2}\right)_{N_{2}-N_{3}} \cdots\left(q^{2} ; q^{2}\right)_{N_{k-1}}} \\
& =\frac{\left(q^{2} ; q^{4}\right)_{\infty}\left(q^{4 k}, q^{2 i-1}, q^{4 k-2 i+1} ; q^{4 k}\right)_{\infty}}{(q ; q)_{\infty}}
\end{aligned}
$$

Bressoud [10] also showed that the left-hand side of (1.3) can be interpreted combinatorially as the generating function of $C_{k, i}(n)$ in Theorem 1.1. More precisely, he gave the following formula for the generating function of $C_{k, i}(m, n)$, where $C_{k, i}(m, n)$ denotes the number of partitions enumerated by $C_{k, i}(n)$ with exactly $m$ parts.

Theorem 1.6 (Bressoud). For $k \geq i \geq 1$,

$$
\begin{aligned}
& \sum_{m, n \geq 0} C_{k, i}(m, n) x^{m} q^{n} \\
& =\sum_{N_{1} \geq N_{2} \geq \cdots \geq N_{k-1} \geq 0} \frac{\left(-q^{1-2 N_{1}} ; q^{2}\right)_{N_{1}} q^{2\left(N_{1}^{2}+\cdots+N_{k-1}^{2}+N_{i}+\cdots+N_{k-1}\right)} x^{N_{1}+\cdots+N_{k-1}}}{\left(q^{2} ; q^{2}\right)_{N_{1}-N_{2}}\left(q^{2} ; q^{2}\right)_{N_{2}-N_{3}} \cdots\left(q^{2} ; q^{2}\right)_{N_{k-1}}} .
\end{aligned}
$$

In recent years, many overpartition analogues of classical partition theorems have been proved, see, for example, Chen, Sang and Shi [12], Corteel and Mallet [13], Corteel, Lovejoy and Mallet [14, and Lovejoy [19,20,22,23]. In particular, Lovejoy [20] obtained an overpartition analogue of the Andrews-Göllnitz-Gordon theorem for $i=k$. In this paper, we give an overpartition analogue of the Andrews-Göllnitz-Gordon theorem in the general case. We also obtain an overpartition analogue of Bressoud's identity (1.3).

Recall that an overpartition of $n$ is a partition of $n$ in which the first occurrence of a number can be overlined. For an overpartition $\lambda$ of $n$, let $f_{t}(\lambda)\left(\operatorname{resp} . f_{\bar{t}}(\lambda)\right)$ be the number of occurrences of $t$ (resp. $\bar{t}$ ) in $\lambda$, without ambiguity, we write $f_{t}$ (resp. $f_{\bar{t}}$ ) for short. By the definition of an overpartition, it is clear to see that $f_{\bar{t}}(\lambda)=0$ or 1 .

We obtain the following overpartition analogue of the Andrews-Göllnitz-Gordon theorem. 
Theorem 1.7. For $k \geq i \geq 1$, let $O_{k, i}(n)$ denote the number of overpartitions of $n$ of the form $\left(\overline{1}^{f_{\overline{1}}}, 1^{f_{1}}, \overline{2}^{f_{\overline{2}}}, 2^{f_{2}}, \ldots\right)$ such that

(1) $f_{\overline{1}}(\lambda)+f_{2}(\lambda) \leq i-1$;

(2) $f_{\overline{2 t}}(\lambda)+f_{2 t}(\lambda)+f_{\overline{2 t+1}}(\lambda)+f_{2 t+2}(\lambda) \leq k-1$;

(3) If $f_{2 t+1}(\lambda) \geq 1$, then $f_{2 t+2}(\lambda) \leq k-2$.

For $k>i \geq 1$, let $P_{k, i}(n)$ denote the number of overpartitions of $n$ with non-overlined parts $\not \equiv 0, \pm(2 i-1) \quad(\bmod 4 k-2)$ and let $P_{k, k}(n)$ denote the number of overpartitions of $n$ into parts not divisible by $2 k-1$.

Then for $k \geq i \geq 1$ and $n \geq 0$,

$$
O_{k, i}(n)=P_{k, i}(n) .
$$

It should be noted that for an overpartition $\lambda$ counted by $O_{k, i}(n)$ without overlined even parts and non-overlined odd parts, if we change overlined odd parts of $\lambda$ to nonoverlined odd parts, then we get a partition counted by $C_{k, i}(n)$. Hence we could say that Theorem 1.7 is an overpartition analogue of Theorem 1.1.

We also obtained the following overpartition analogue of Bressoud's identity (1.3), which can be viewed as the corresponding generating function version of Theorem 1.7 .

Theorem 1.8. For $k \geq i \geq 1$,

$$
\begin{aligned}
& \sum_{N_{1} \geq \cdots \geq N_{k-1} \geq 0} \frac{\left(-q^{2-2 N_{1}} ; q^{2}\right)_{N_{1}-1}\left(-q^{1-2 N_{1}} ; q^{2}\right)_{N_{1}} q^{2\left(N_{1}^{2}+\cdots+N_{k-1}^{2}+N_{i+1}+\cdots+N_{k-1}\right)}\left(1+q^{2 N_{i}}\right)}{\left(q^{2} ; q^{2}\right)_{N_{1}-N_{2}} \cdots\left(q^{2} ; q^{2}\right)_{N_{k-2}-N_{k-1}}\left(q^{2} ; q^{2}\right)_{N_{k-1}}} \\
& =\frac{(-q ; q)_{\infty}\left(q^{2 i-1}, q^{4 k-1-2 i}, q^{4 k-2} ; q^{4 k-2}\right)_{\infty}}{(q ; q)_{\infty}} .
\end{aligned}
$$

We will first prove Theorem 1.8 by using Bailey's lemma and a change of base formula due to Bressoud, Ismail and Stanton [11. We then use Theorem 1.8 to derive Theorem 1.7. More precisely, let $O_{k, i}(m, n)$ denote the number of overpartitions counted by $O_{k, i}(n)$ with exactly $m$ parts, we shall give a combinatorial proof of the following formula for the generating function of $O_{k, i}(m, n)$ by introducing the Göllnitz-Gordon marking of an overpartition.

Theorem 1.9. For $k \geq i \geq 1$,

$$
\begin{aligned}
& \sum_{m, n \geq 0} O_{k, i}(m, n) x^{m} q^{n} \\
& =\sum_{N_{1} \geq \cdots \geq N_{k-1} \geq 0} \frac{\left(-q^{2-2 N_{1}} ; q^{2}\right)_{N_{1}-1}\left(-q^{1-2 N_{1}} ; q^{2}\right)_{N_{1}}}{\left(q^{2} ; q^{2}\right)_{N_{1}-N_{2}} \cdots} \\
& \quad \times \frac{q^{2\left(N_{1}^{2}+\cdots+N_{k-1}^{2}+N_{i+1}+\cdots+N_{k-1}\right)}\left(1+q^{2 N_{i}}\right) x^{N_{1}+\cdots+N_{k-1}}}{\left(q^{2} ; q^{2}\right)_{N_{k-2}-N_{k-1}}\left(q^{2} ; q^{2}\right)_{N_{k-1}}} .
\end{aligned}
$$


Setting $x=1$ in (1.6), we obtain the generating function for $O_{k, i}(n)$ which is the left-hand side of (1.5). On the other hand, it is evident that the generating function of $P_{k, i}(n)$ equals

$$
\sum_{n \geq 0} P_{k, i}(n) q^{n}=\frac{(-q ; q)_{\infty}\left(q^{2 i-1}, q^{4 k-2 i-1}, q^{4 k-2} ; q^{4 k-2}\right)_{\infty}}{(q ; q)_{\infty}}
$$

which is the right-hand side of (1.5). Hence we are led to Theorem 1.7 by Theorem 1.8 ,

The paper is organized as follows. In Section 2, we first review some necessary results on Bailey pairs and then give a proof of Theorem 1.8 by combining Bailey's lemma and a change of base formula. In Section 3, we begin with introducing the notion of the Göllnitz-Gordon marking of an overpartition and then give an outline of the proof of the formula for the generating function of $O_{k, i}(m, n)$ in Theorem 1.9. It turns out that the proof of Theorem 1.9 reduces to the proofs of two relations stated in Lemma 3.5 and Lemma 3.6, respectively. Section 4 and Section 5 are devoted to the bijective proofs of these two relations respectively. In Section 6, we complete the proof of Theorem 1.9.

\section{Proof of Theorem 1.8}

We first give a brief review of some relevant results on Bailey pairs required in the proof of Theorem 1.8. For more on Bailey pairs, see, for example, [1, 1, 8, 11, 21, 24, 26]. Recall that a pair of sequences $\left(\alpha_{n}(a, q), \beta_{n}(a, q)\right)$ is called a Bailey pair with parameters $(a, q)$ (or a Bailey pair for short) if for all $n \geq 0$,

$$
\beta_{n}(a, q)=\sum_{r=0}^{n} \frac{\alpha_{r}(a, q)}{(q ; q)_{n-r}(a q ; q)_{n+r}} .
$$

Bailey's lemma was first given by Bailey [9] and was formulated by Andrews [6, 7] in the following form.

Theorem 2.1 (Bailey's lemma). If $\left(\alpha_{n}(a, q), \beta_{n}(a, q)\right)$ is a Bailey pair, then $\left(\alpha_{n}^{\prime}(a, q), \beta_{n}^{\prime}(a, q)\right)$ is also a Bailey pair, where

$$
\begin{aligned}
& \alpha_{n}^{\prime}(a, q)=\frac{\left(\rho_{1} ; q\right)_{n}\left(\rho_{2} ; q\right)_{n}}{\left(a q / \rho_{1} ; q\right)_{n}\left(a q / \rho_{2} ; q\right)_{n}}\left(\frac{a q}{\rho_{1} \rho_{2}}\right)^{n} \alpha_{n}(a, q), \\
& \beta_{n}^{\prime}(a, q)=\sum_{j=0}^{n} \frac{\left(\rho_{1} ; q\right)_{j}\left(\rho_{2} ; q\right)_{j}\left(a q / \rho_{1} \rho_{2} ; q\right)_{n-j}}{\left(a q / \rho_{1} ; q\right)_{n}\left(a q / \rho_{2} ; q\right)_{n}(q ; q)_{n-j}}\left(\frac{a q}{\rho_{1} \rho_{2}}\right)^{j} \beta_{j}(a, q) .
\end{aligned}
$$

Andrews first noticed that Bailey's lemma can create a new Bailey pair from a given one. Hence iterating Theorem 2.1 produces a sequence of Bailey pairs, which has been called a Bailey chain. Based on this observation, Andrews [6] showed that the AndrewsGordon identity (1.1) in Theorem 1.3 holds for $i=1$ and $i=k$ by iteratively using the following specialization of Bailey's lemma. 
Lemma $2.2\left(\rho_{1}, \rho_{2} \rightarrow \infty\right.$ in Theorem 2.1). If $\left(\alpha_{n}(a, q), \beta_{n}(a, q)\right)$ is a Bailey pair, then $\left(\alpha_{n}^{\prime}(a, q), \beta_{n}^{\prime}(a, q)\right)$ is also a Bailey pair, where

$$
\begin{aligned}
& \alpha_{n}^{\prime}(a, q)=a^{n} q^{n^{2}} \alpha_{n}(a, q), \\
& \beta_{n}^{\prime}(a, q)=\sum_{j=0}^{n} \frac{a^{j} q^{j^{2}}}{(q ; q)_{n-j}} \beta_{j}(a, q) .
\end{aligned}
$$

Subsequently, Agarwal, Andrews and Bressoud [1] gave an extension of a Bailey chain known as a Bailey lattice and used the Bailey lattice to prove the Andrews-Gordon identity (1.1) holds for $1 \leq i \leq k$. Bressoud, Ismail and Stanton [11] provided an alternative proof of this identity by combining Bailey's lemma with the following proposition.

Proposition 2.3. [11, Proposition 4.1] If $\left(\alpha_{n}(1, q), \beta_{n}(1, q)\right)$ is a Bailey pair, where

$$
\alpha_{n}(1, q)= \begin{cases}1, & \text { for } n=0 \\ (-1)^{n} q^{A n^{2}}\left(q^{(A-1) n}+q^{-(A-1) n}\right), & \text { for } n \geq 1\end{cases}
$$

then $\left(\alpha_{n}^{\prime}(1, q), \beta_{n}^{\prime}(1, q)\right)$ is also a Bailey pair, where

$$
\begin{aligned}
& \alpha_{n}^{\prime}(1, q)= \begin{cases}1, & \text { for } n=0, \\
(-1)^{n} q^{A n^{2}}\left(q^{A n}+q^{-A n}\right), & \text { for } n \geq 1,\end{cases} \\
& \beta_{n}^{\prime}(1, q)=q^{n} \beta_{n}(1, q) .
\end{aligned}
$$

By iteratively using Bailey's lemma and Proposition 2.3, Bressoud, Ismail and Stanton [11] also provided a proof of Bressoud's identity (1.3) in Theorem 1.5. Moreover, they established new versions of Bailey's lemma, known as change of base formulas, which change the base in Bailey pairs from $q$ to $q^{2}$ or $q^{3}$. Iterating these change of base formulas, they obtained many new multisum Rogers-Ramanujan identities.

To prove Theorem 1.8, we shall make use of the following special case of the change of base formula due to Bressoud, Ismail and Stanton.

Lemma 2.4. [11, Theorem 2.5, $B \rightarrow \infty$ ] If $\left(\alpha_{n}(a, q), \beta_{n}(a, q)\right)$ is a Bailey pair, then $\left(\alpha_{n}^{\prime}(a, q), \beta_{n}^{\prime}(a, q)\right)$ is also a Bailey pair, where

$$
\begin{aligned}
& \alpha_{n}^{\prime}(a, q)=\frac{1+a}{1+a q^{2 n}} q^{n} \alpha_{n}\left(a^{2}, q^{2}\right), \\
& \beta_{n}^{\prime}(a, q)=\sum_{k=0}^{n} \frac{(-a ; q)_{2 k} q^{k}}{\left(q^{2} ; q^{2}\right)_{n-k}} \beta_{k}\left(a^{2}, q^{2}\right) .
\end{aligned}
$$

We are now in a position to prove Theorem 1.8 . 
Proof of Theorem 1.8: We begin with the unit Bailey pair [25, H(17)],

$$
\begin{aligned}
& \alpha_{n}^{(0)}(1, q)= \begin{cases}1, & \text { if } n=0, \\
(-1)^{n} q^{n^{2} / 2}\left(q^{-n / 2}+q^{n / 2}\right), & \text { if } n \geq 1,\end{cases} \\
& \beta_{n}^{(0)}(1, q)= \begin{cases}1, & \text { if } n=0, \\
0, & \text { if } n \geq 1 .\end{cases}
\end{aligned}
$$

Substituting (2.4) into Lemma 2.2, we get a new Bailey pair $\left(\alpha_{n}^{(1)}(1, q), \beta_{n}^{(1)}(1, q)\right)$,

$$
\begin{aligned}
& \alpha_{n}^{(1)}(1, q)= \begin{cases}1, & \text { if } n=0 \\
(-1)^{n} q^{3 n^{2} / 2}\left(q^{-n / 2}+q^{n / 2}\right), & \text { if } n \geq 1,\end{cases} \\
& \beta_{n}^{(1)}(1, q)=\frac{1}{(q ; q)_{n}} .
\end{aligned}
$$

Alternatively applying Proposition 2.3 and Lemma 2.2 $k-i-1$ times to (2.5) yields the following Bailey pair $\left(\alpha_{n}^{(2 k-2 i-1)}(1, q), \beta_{n}^{(2 k-2 i-1)}(1, q)\right)$,

$$
\begin{aligned}
& \alpha_{n}^{(2 k-2 i-1)}(1, q)= \begin{cases}1, & \text { if } n=0, \\
(-1)^{n} q^{\frac{2 k-2 i+1}{2} n^{2}}\left(q^{-\frac{2 k-2 i-1}{2} n}+q^{\frac{2 k-2 i-1}{2} n}\right), & \text { if } n \geq 1\end{cases} \\
& \beta_{n}^{(2 k-2 i-1)}(1, q)=\sum_{n \geq N_{i+1} \geq \cdots \geq N_{k-1} \geq 0} \frac{q^{N_{i+1}^{2}+N_{i+2}^{2}+\cdots+N_{k-1}^{2}+N_{i+1}+\cdots+N_{k-1}}}{(q ; q)_{n-N_{i+1}}(q ; q)_{N_{i+1}-N_{i+2}} \cdots(q ; q)_{N_{k-2}-N_{k-1}}(q ; q)_{N_{k-1}}} .
\end{aligned}
$$

Applying Proposition 2.3 to (2.6) gives the Bailey pair $\left(\alpha_{n}^{(2 k-2 i)}(1, q), \beta_{n}^{(2 k-2 i)}(1, q)\right)$,

$$
\begin{aligned}
& \alpha_{n}^{(2 k-2 i)}(1, q)= \begin{cases}1, & \text { if } n=0, \\
(-1)^{n} q^{\frac{2 k-2 i+1}{2} n^{2}}\left(q^{-\frac{2 k-2 i+1}{2} n}+q^{\frac{2 k-2 i+1}{2} n}\right), & \text { if } n \geq 1,\end{cases} \\
& \beta_{n}^{(2 k-2 i)}(1, q)=q^{n} \sum_{n \geq N_{i+1} \geq \cdots \geq N_{k-1} \geq 0} \frac{q^{N_{i+1}^{2}+N_{i+2}^{2}+\cdots+N_{k-1}^{2}+N_{i+1}+\cdots+N_{k-1}}}{(q ; q)_{n-N_{i+1}}(q ; q)_{N_{i+1}-N_{i+2}} \cdots(q ; q)_{N_{k-2}-N_{k-1}}(q ; q)_{N_{k-1}}} .
\end{aligned}
$$

By the definition of a Bailey pair, it is easy to see that the sum of (2.6) and (2.7) generates a new Bailey pair $\left(\alpha_{n}^{(2 k-2 i+1)}(1, q), \beta_{n}^{(2 k-2 i+1)}(1, q)\right)$,

$$
\begin{aligned}
& \alpha_{n}^{(2 k-2 i+1)}(1, q)= \begin{cases}1, & \text { if } n=0, \\
(-1)^{n} q^{\frac{2 k-2 i+1}{2} n^{2}}\left(q^{-\frac{2 k-2 i+1}{2} n}+q^{\frac{2 k-2 i-1}{2} n}\right)\left(1+q^{n}\right) / 2, & \text { if } n \geq 1,\end{cases} \\
& \beta_{n}^{(2 k-2 i+1)}(1, q)=\sum_{n \geq N_{i+1} \geq \cdots \geq N_{k-1} \geq 0} \frac{\left(1+q^{n}\right) q^{N_{i+1}^{2}+N_{i+2}^{2}+\cdots+N_{k-1}^{2}+N_{i+1}+\cdots+N_{k-1}}}{2(q ; q)_{n-N_{i+1}}(q ; q)_{N_{i+1}-N_{i+2}} \cdots(q ; q)_{N_{k-2}-N_{k-1}}(q ; q)_{N_{k-1}}} .
\end{aligned}
$$


Applying Lemma 2.2 to (2.8) $i-1$ times, we get the Bailey pair $\left(\alpha_{n}^{(2 k-i)}(1, q), \beta_{n}^{(2 k-i)}(1, q)\right)$,

$$
\begin{aligned}
& \alpha_{n}^{(2 k-i)}(1, q)= \begin{cases}1, & \text { if } n=0, \\
(-1)^{n} q^{\frac{2 k-1}{2} n^{2}}\left(q^{-\frac{2 k-2 i+1}{2} n}+q^{\frac{2 k-2 i-1}{2} n}\right)\left(1+q^{n}\right) / 2, & \text { if } n \geq 1,\end{cases} \\
& \beta_{n}^{(2 k-i)}(1, q)=\sum_{n \geq N_{2} \geq \cdots \geq N_{k-1} \geq 0} \frac{q^{N_{2}^{2}+N_{3}^{2}+\cdots+N_{k-1}^{2}+N_{i+1}+\cdots+N_{k-1}}\left(1+q^{N_{i}}\right)}{2(q ; q)_{n-N_{2}}(q ; q)_{N_{2}-N_{3}} \cdots(q ; q)_{N_{k-2}-N_{k-1}}(q ; q)_{N_{k-1}}} .
\end{aligned}
$$

Plugging (2.9) into Lemma 2.4, we get a Bailey pair $\left(\alpha_{n}^{(2 k-i+1)}(1, q), \beta_{n}^{(2 k-i+1)}(1, q)\right)$,

$$
\begin{aligned}
& \alpha_{n}^{(2 k-i+1)}(1, q)= \begin{cases}1, & \text { if } n=0, \\
(-1)^{n} q^{(2 k-1) n^{2}}\left(q^{-2(k-i) n}+q^{2(k-i) n}\right), & \text { if } n \geq 1,\end{cases} \\
& \beta_{n}^{(2 k-i+1)}(1, q)=\sum_{n \geq N_{1} \geq \cdots \geq N_{k-1} \geq 0} \frac{(-q ; q)_{2 N_{1}-1} q^{N_{1}+2\left(N_{2}^{2}+N_{3}^{2}+\cdots+N_{k-1}^{2}+N_{i+1}+\cdots+N_{k-1}\right)}\left(1+q^{2 N_{i}}\right)}{\left(q^{2} ; q^{2}\right)_{n-N_{1}}\left(q^{2} ; q^{2}\right)_{N_{1}-N_{2}} \cdots\left(q^{2} ; q^{2}\right)_{N_{k-2}-N_{k-1}}\left(q^{2} ; q^{2}\right)_{N_{k-1}}} .
\end{aligned}
$$

By the definition of a Bailey pair, letting $n \rightarrow \infty$ and multiplying both sides by $\left(q^{2} ; q^{2}\right)_{\infty}$, we obtain

$$
\begin{aligned}
& \sum_{N_{1} \geq \cdots \geq N_{k-1} \geq 0} \frac{(-q ; q)_{2 N_{1}-1} q^{N_{1}+2\left(N_{2}^{2}+N_{3}^{2}+\cdots+N_{k-1}^{2}+N_{i+1}+\cdots+N_{k-1}\right)}\left(1+q^{2 N_{i}}\right)}{\left(q^{2} ; q^{2}\right)_{N_{1}-N_{2}}\left(q^{2} ; q^{2}\right)_{N_{2}-N_{3}} \cdots\left(q^{2} ; q^{2}\right)_{N_{k-2}-N_{k-1}}\left(q^{2} ; q^{2}\right)_{N_{k-1}}} \\
= & \frac{(-q ; q)_{\infty}}{(q ; q)_{\infty}}\left(1+\sum_{n=1}^{\infty}(-1)^{n} q^{(2 k-1) n^{2}}\left(q^{-2(k-i) n}+q^{2(k-i) n}\right)\right) .
\end{aligned}
$$

Using Jacobi's triple product identity, we see that

$$
1+\sum_{n=1}^{\infty}(-1)^{n} q^{(2 k-1) n^{2}}\left(q^{-2(k-i) n}+q^{2(k-i) n}\right)=\left(q^{2 i-1}, q^{4 k-2 i-1}, q^{4 k-2} ; q^{4 k-2}\right)_{\infty} .
$$

Submitting (2.11) into (2.10), and noting that

$$
(-q ; q)_{2 N_{1}-1} q^{N_{1}}=\left(-q^{2-2 N_{1}} ; q^{2}\right)_{N_{1}-1}\left(-q^{1-2 N_{1}} ; q^{2}\right)_{N_{1}} q^{2 N_{1}^{2}}
$$

we obtain (1.5). Thus we complete the proof of Theorem 1.8.

\section{The Göllnitz-Gordon marking of an overpartition}

In this section, we first introduce the Göllnitz-Gordon marking of an overpartition and then give an outline of the proof of Theorem 1.9.

Kurşungöz [18] introduced the notion of the Gordon marking of an ordinary partition and gave a combinatorial proof of Theorem 1.4. The Gordon marking of an ordinary 
partition $\eta$ is an assignment of positive integers (marks) to the parts of $\eta$ from smallest to largest such that the marks are as small as possible subject to the condition that equal or consecutive parts are assigned different marks. More precisely, let $\eta=\left(\eta_{1}, \eta_{2}, \ldots, \eta_{\ell}\right)$ where $1 \leq \eta_{1} \leq \eta_{2} \leq \cdots \leq \eta_{\ell}$. Assign 1 to $\eta_{1}$. For $p>1$, assume that $s$ is the least positive integer that is not used to mark the parts $\eta_{j}$ with $\eta_{p}-\eta_{j} \leq 1$ for $j<p$. Then, we assign $s$ to $\eta_{p}$. For example, the Gordon marking of $\eta=(1,1,2,2,2,3,4,5,5,6,6,6)$ is

$$
G(\eta)=\left(1_{1}, 1_{2}, 2_{3}, 2_{4}, 2_{5}, 3_{1}, 4_{2}, 5_{1}, 5_{3}, 6_{2}, 6_{4}, 6_{5}\right) .
$$

The Gordon marking of an ordinary partition can also be represented by an array where the column indicates the value of parts and the row (counted from bottom to top) indicates the mark listed outside the brackets, so the Gordon marking of $\eta$ above can be represented as:

$$
G(\eta)=\left[\begin{array}{rrrrrrr} 
& 2 & & & & 6 \\
& 2 & & & & 6 \\
& 2 & & & 5 & \\
1 & & & 4 & & 6 \\
1 & & 3 & & 5 &
\end{array}\right] \begin{aligned}
& 5 \\
& 4 \\
& 3 \\
& 2 \\
& 1
\end{aligned}
$$

We call this array the Gordon marking representation of an ordinary partition. Let $N_{r}$ denote the number of parts in the $r$-th row of the Gordon marking representation of an ordinary partition $\eta$, that is, the number of $r$-marked parts in the Gordon marking of $\eta$. From the definition of the Gordon marking of an ordinary partition, it is not difficult to see that $N_{1} \geq N_{2} \geq \cdots$. Furthermore, if $\eta$ is counted by $B_{k, i}(n)$ in Theorem 1.2, then there are no $k$ or greater marked parts in the Gordon marking of $\eta$, namely, there are at most $k-1$ rows in the Gordon marking representation of $\eta$.

Let $\mathbb{B}_{N_{1}, \ldots, N_{k-1} ; i}(n)$ denote the set of partitions $\eta$ counted by $B_{k, i}(n)$ such that there are $N_{r}$ parts in the $r$-th row of the Gordon marking representation of $\eta$ for $1 \leq r \leq k-1$. Define

$$
\mathbb{B}_{N_{1}, \ldots, N_{k-1} ; i}=\bigcup_{n \geq 0} \mathbb{B}_{N_{1}, \ldots, N_{k-1} ; i}(n) .
$$

Kurşungöz [18] established the following identity by introducing backward and forward moves defined on the Gordon marking of an ordinary partition.

$$
\sum_{\eta \in \mathbb{B}_{N_{1}, \ldots, N_{k-1} ; i}} q^{|\eta|}=\frac{q^{N_{1}^{2}+\cdots+N_{k-1}^{2}+N_{i}+\cdots+N_{k-1}}}{(q ; q)_{N_{1}-N_{2}} \cdots(q ; q)_{N_{k-2}-N_{k-1}}(q ; q)_{N_{k-1}}}
$$

where $|\eta|$ denotes the sum of parts of $\eta$.

Let $\mathbb{B}_{k, i}(m, n)$ denote the set of partitions counted by $B_{k, i}(m, n)$ in Theorem 1.4. From the definition of $\mathbb{B}_{N_{1}, \ldots, N_{k-1} ;}(n)$, we see that

$$
\mathbb{B}_{k, i}(m, n)=\bigcup_{\substack{N_{1} \geq \cdots \geq N_{k-1} \geq 0 \\ N_{1}+\cdots+N_{k-1}=m}} \mathbb{B}_{N_{1}, \ldots, N_{k-1} ; i}(n),
$$


SO

$$
\sum_{m, n \geq 0} B_{k, i}(m, n) x^{m} q^{n}=\sum_{N_{1} \geq N_{2} \geq \ldots \geq N_{k-1} \geq 0} x^{N_{1}+N_{2}+\ldots+N_{k-1}} \sum_{\eta \in \mathbb{B}_{N_{1}, \ldots, N_{k-1} ; i}} q^{|\eta|} .
$$

Therefore, inserting (3.1) into (3.2) to give rise to Theorem 1.4,

To show Theorem 1.9, we introduce the Göllnitz-Gordon marking of an overpartition which is different from the Gordon marking of an overpartition introduced by Chen, Sang and Shi [12. It should be mentioned that an ordinary partition can be marked with Göllnitz-Gordon marking, but the Göllnitz-Gordon marking of an ordinary partition is different from the Gordon marking of an ordinary partition.

In the remainder of this paper, we write an overpartition $\lambda$ as the form $\left(\lambda_{1}, \lambda_{2}, \ldots, \lambda_{\ell}\right)$ where $\lambda_{1} \leq \lambda_{2} \leq \cdots \leq \lambda_{\ell}$ are ranked in the following order,

$$
\overline{1}<1<\overline{2}<2<\cdots .
$$

The $\lambda_{j}$ is called the $j$-th part of an overpartition $\lambda$. Denote the size of $\lambda_{j}$ by $\left|\lambda_{j}\right|$. If $\left|\lambda_{j}\right|=a_{j}$, then we write $\lambda_{j}=\overline{a_{j}}$ to indicate that $\lambda_{j}$ is an overlined part and write $\lambda_{j}=a_{j}$ to indicate that $\lambda_{j}$ is a non-overlined part.

Definition 3.1 (Göllnitz-Gordon marking). The Göllnitz-Gordon marking of an overpartition $\lambda$ is an assignment of positive integers (marks) to parts of $\lambda=\left(\lambda_{1}, \lambda_{2}, \ldots, \lambda_{\ell}\right)$ from smallest to largest. Assign 1 to $\lambda_{1}$. For $p>1$, assume that the part $\lambda_{j}$ has been assigned a mark for $j<p$. We consider the following four cases:

(1) If $\lambda_{p}$ is a non-overlined odd part, then assign 1 to $\lambda_{p}$.

(2) If $\lambda_{p}$ is an overlined even part, then assign 1 to $\lambda_{p}$.

(3) If $\lambda_{p}$ is an overlined odd part, and let $s$ be the least positive integer that is not used to mark the parts $\lambda_{j}$ with $\left|\lambda_{p}\right|-\left|\lambda_{j}\right|=1$ for $j<p$, then assign $s$ to $\lambda_{p}$.

(4) If $\lambda_{p}$ is a non-overlined even part, say $\lambda_{p}=2 t+2$, and define

$\diamond f$ to be the least positive integer that is not used to mark the parts $\lambda_{j}$ with $\left|\lambda_{p}\right|-\left|\lambda_{j}\right| \leq$ 2 for $j<p$,

$\diamond g$ to be the least positive integer that has been used to mark the parts $\lambda_{j}$ with $\left|\lambda_{p}\right|-$ $\left|\lambda_{j}\right|=2$ for $j<p$. If such $\lambda_{j}$ does not occur in $\lambda$, then set $g=0$,

then we may assign $f$ or $g$ to $\lambda_{p}$ by considering the following two subcases:

(4.1) If $\lambda$ satisfies four conditions simultaneously: (i) $g \geq 2$; (ii) the mark of $\lambda_{p-1}$ is $g-1$; (iii) $2 t+1$ or $\overline{2 t+2}$ occurs in $\lambda$; (iv) $\overline{2 t+1}$ does not occur in $\lambda$, then assign $g$ to $\lambda_{p}$;

(4.2) Otherwise, assign $f$ to $\lambda_{p}$. 
For example, we consider the overpartition

$$
\begin{aligned}
& \lambda_{1}, \quad \lambda_{2}, \quad \lambda_{3}, \quad \lambda_{4}, \quad \lambda_{5}, \quad \lambda_{6}, \quad \lambda_{7}, \quad \lambda_{8}, \quad \lambda_{9}, \quad \lambda_{10} \\
& \downarrow \begin{array}{lllllllllllll} 
& \downarrow & \downarrow & \downarrow & \downarrow & \downarrow & \downarrow & \downarrow & \downarrow & \downarrow & \downarrow
\end{array} \\
& \lambda=\left(\begin{array}{lllllllllll}
1, & 1, & \overline{2} & 2 & 2 & \overline{3}, & \overline{4}, & 6 & 7, & 8, & 8
\end{array}\right) \text {. }
\end{aligned}
$$

By Definition 3.1, we see that $\lambda_{1}=1, \lambda_{2}=1$ and $\lambda_{8}=7$ should be marked with 1 since they are non-overlined odd parts. On the other hand, $\lambda_{3}=\overline{2}$ and $\lambda_{6}=\overline{4}$ are overlined even parts, so they are also marked with 1 . Hence, we have

$$
\begin{aligned}
& \begin{array}{llllllllll}
\lambda_{1}, & \lambda_{2}, & \lambda_{3}, & \lambda_{4}, & \lambda_{5}, & \lambda_{6}, & \lambda_{7}, & \lambda_{8}, & \lambda_{9}, & \lambda_{10}
\end{array}
\end{aligned}
$$

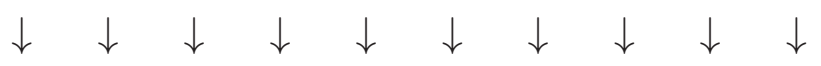

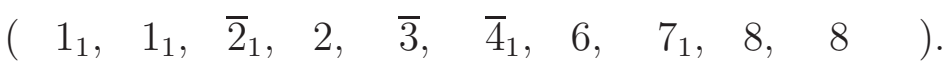

The part $\lambda_{4}=2$ should be marked with 2 since it is a non-overlined even part and it does not satisfy the conditions (4.1) in Definition 3.1. The part $\lambda_{5}=\overline{3}$ is marked with 3 since it is an overlined odd part and $\lambda_{3}=\overline{2}$ and $\lambda_{4}=2$ are marked with 1 and 2 respectively. The part $\lambda_{7}=6$ is marked with 2 since it is a non-overlined even part and it does not satisfy the conditions (4.1) in Definition 3.1. The part $\lambda_{9}=8$ is marked with 2 since it is a non-overlined even part and it satisfies the conditions (4.1) in Definition 3.1. The part $\lambda_{10}=8$ is marked with 3 since it is a non-overlined even part and it does not satisfy the conditions (4.1) in Definition 3.1. So the Göllnitz-Gordon marking of $\lambda$ is

$$
G G(\lambda)=\left(1_{1}, 1_{1}, \overline{2}_{1}, 2_{2}, \overline{3}_{3}, \overline{4}_{1}, 6_{2}, 7_{1}, 8_{2}, 8_{3}\right) .
$$

It can also be represented by an array where column indicates the size of parts, and the row (counted from bottom to top) indicates the mark listed outside the brackets, so the Göllnitz-Gordon marking of $\lambda$ above would be

$$
G G(\lambda)=\left[\begin{array}{lllllll} 
& & \overline{3} & & & & 8 \\
& 2 & & & 6 & & 8 \\
1^{2} & \overline{2} & & \overline{4} & & 7 &
\end{array}\right] \begin{aligned}
& 3 \\
& 2 \\
& 1
\end{aligned} .
$$

Similarly, we will call this diagram the Göllnitz-Gordon marking representation of an overpartition. Note that non-overlined odd parts would be repeated in the first row of the Göllnitz-Gordon marking representation of $\lambda$, so for $t \geq 2$, we will use $(2 j+1)^{t}$ to denote that there are $t(2 j+1)$ 's in the first row of the Göllnitz-Gordon marking representation of $\lambda$, and $\overline{2 j+1}^{t}$ to denote that there are a $\overline{2 j+1}$ and $t-1(2 j+1)$ 's in the first row of the Göllnitz-Gordon marking representation of $\lambda$.

Let $N_{r}$ denote the number of parts in the $r$-th row of the Göllnitz-Gordon marking representation of an overpartition. From the definition of Göllnitz-Gordon marking, it is not hard to show that $N_{1} \geq N_{2} \geq \cdots$. For the example above, we have $N_{1}=5, N_{2}=3$, and $N_{3}=2$. 
From the definition of Göllnitz-Gordon marking, we see that if $\lambda$ is counted by $O_{k, i}(n)$ in Theorem 1.7, then $f_{\overline{1}}(\lambda)+f_{2}(\lambda) \leq i-1$ and there are no $k$ or greater marked parts in the the Göllnitz-Gordon marking of $\lambda$, that is, there are at most $k-1$ rows in the Göllnitz-Gordon marking representation of $\lambda$, and vice versa. More precisely, we have following proposition.

Proposition 3.2. For $k \geq i \geq 1$, an overpartition $\lambda$ is counted by $O_{k, i}(n)$ if and only if the number of occurrences of $\overline{1}$ and 2 in $\lambda$ is not exceed $i-1$ and there are at most $k-1$ rows in the Göllnitz-Gordon marking representation of $\lambda$.

It should be noted that for the parts $2 t+2$ in $\lambda$, if $f_{\overline{2 t+1}}(\lambda)=0, f_{\overline{2 t}}(\lambda)=0$ and

$$
f_{2 t}(\lambda)+f_{2 t+2}(\lambda)=k-1,
$$

and the least positive integer $q$ that has been used to mark the parts $2 t$ in $\lambda$ is greater than 1 , and there is at least one $2 t+1$ or $\overline{2 t+2}$ in $\lambda$ which will be marked with 1 , then the marks of $2 t+2$ will be less than $k$ since there is a $2 t+2$ in $\lambda$ marked with $g$ which satisfies the conditions in (4.1) of Definition 3.1. This is the reason that the marking of non-overlined even parts in the definition of Göllnitz-Gordon marking is more complicated.

For $k \geq i \geq 1$, let $\mathbb{O}_{k, i}(m, n)$ denote the set of overpartitions counted by $O_{k, i}(m, n)$, we will classify $\mathbb{O}_{k, i}(m, n)$ by considering whether the smallest part of an overpartition in $\mathbb{O}_{k, i}(m, n)$ is a non-overlined odd part or an overlined even part. Note that the parts of an overpartition are ordered by (3.3) . Let $\mathbb{F}_{k, i}(m, n)$ denote the set of overpartitions in $\mathbb{O}_{k, i}(m, n)$ for which the smallest part is an overlined odd part or a non-overlined even part, and let $\mathbb{H}_{k, i}(m, n)$ denote the set of overpartitions in $\mathbb{O}_{k, i}(m, n)$ with the smallest part being a non-overlined odd part or an overlined even part. Obviously,

$$
\mathbb{O}_{k, i}(m, n)=\mathbb{F}_{k, i}(m, n) \cup \mathbb{H}_{k, i}(m, n) .
$$

Let $F_{k, i}(m, n)=\left|\mathbb{F}_{k, i}(m, n)\right|$ and $H_{k, i}(m, n)=\left|\mathbb{H}_{k, i}(m, n)\right|$. Then

$$
O_{k, i}(m, n)=F_{k, i}(m, n)+H_{k, i}(m, n) .
$$

There is a relation between $F_{k, i}(m, n)$ and $H_{k, i}(m, n)$.

Lemma 3.3. For $k \geq i \geq 2$,

$$
F_{k, i}(m, n)=H_{k, i-1}(m, n) .
$$

For $k \geq 1$,

$$
F_{k, 1}(m, n)=H_{k, k}(m, n-2 m) .
$$

Proof. For $k \geq i \geq 2$, there is a simple bijection between $\mathbb{F}_{k, i}(m, n)$ and $\mathbb{H}_{k, i-1}(m, n)$. Let $\sigma$ be an overpartition in $\mathbb{F}_{k, i}(m, n)$, we consider two cases: If the smallest part of $\sigma$ is an 
overlined odd part, say $\overline{2 t+1}$, then change it to a non-overlined odd part $2 t+1$. If the smallest part of $\sigma$ is a non-overlined even part, say $2 t$, then change the first $2 t$ of $\sigma$ to an overlined even part $\overline{2 t}$. In either case, we obtain an overpartition $\pi$ in $\mathbb{H}_{k, i-1}(m, n)$. Furthermore, it is easy to see that this process is reversible, and so this map is a bijection. Hence (3.7) holds for $k \geq i \geq 2$.

For $k \geq 1$, we will give a bijection between $\mathbb{F}_{k, 1}(m, n)$ and $\mathbb{H}_{k, k}(m, n-2 m)$. For an overpartition $\sigma \in \mathbb{F}_{k, 1}(m, n)$, by the definition of $\mathbb{F}_{k, 1}(m, n)$, we see that $\sigma$ has $m$ parts and the size of each part of $\sigma$ is greater than 2. There are two cases: If the smallest part of $\sigma$ is an overlined odd part, say $\overline{2 t+1}$, where $t \geq 1$, then change it to a non-overlined odd part $2 t+1$. If the smallest part of $\sigma$ is a non-overlined even part, then change one of the smallest parts, say $2 t$, where $t \geq 2$ to an overlined even part $\overline{2 t}$. In either case, we obtained a new overpartition $\rho$ for which the size of each part is greater than 2 . We then subtract 2 from each part of $\rho$ to obtain the resulting overpartition $\pi$ in $\mathbb{H}_{k, k}(m, n-2 m)$. It is evident to see that this process is reversible, and so this map is a bijection. Hence we arrive at (3.8). This completes the proof.

Using the relation (3.6), it is easy to find that the generating function of $O_{k, i}(m, n)$ can be deduced from the generating functions of $F_{k, i}(m, n)$ and $H_{k, i}(m, n)$. In light of Lemma 3.3. we see that the generating function of $H_{k, i}(m, n)$ can be obtained from the generating function of $F_{k, i}(m, n)$. Hence, it suffices to derive the following generating function of $F_{k, i}(m, n)$ in order to prove Theorem 1.9.

Theorem 3.4. For $k \geq i \geq 1$,

$$
\begin{aligned}
& \sum_{m, n \geq 0} F_{k, i}(m, n) x^{m} q^{n} \\
& =\sum_{N_{1} \geq \cdots \geq N_{k-1} \geq 0} \frac{\left(-q^{2-2 N_{1}} ; q^{2}\right)_{N_{1}-1}\left(-q^{1-2 N_{1}} ; q^{2}\right)_{N_{1}} q^{2\left(N_{1}^{2}+\cdots+N_{k-1}^{2}+N_{i}+\cdots+N_{k-1}\right)} x^{N_{1}+\cdots+N_{k-1}}}{\left(q^{2} ; q^{2}\right)_{N_{1}-N_{2}} \cdots\left(q^{2} ; q^{2}\right)_{N_{k-2}-N_{k-1}}\left(q^{2} ; q^{2}\right)_{N_{k-1}}} .
\end{aligned}
$$

In this paper, we will give a combinatorial proof of Theorem 3.4 based on the GöllnitzGordon marking of an overpartition. Let $\mathbb{F}_{N_{1}, \ldots, N_{k-1} ; i}(n)$ denote the set of overpartitions $\lambda$ in $\mathbb{F}_{k, i}(m, n)$ such that there are $N_{r} r$-marked parts in the Göllnitz-Gordon marking of $\lambda$ for $1 \leq r \leq k-1$.

Set

$$
\mathbb{F}_{N_{1}, \ldots, N_{k-1} ; i}=\bigcup_{n \geq 0} \mathbb{F}_{N_{1}, \ldots, N_{k-1} ; i}(n)
$$

From the definition of $\mathbb{F}_{N_{1}, \ldots, N_{k-1} ; i}$, it is evident to see that

$$
\mathbb{F}_{k, i}(m, n)=\bigcup_{\substack{N_{1} \geq \cdots \geq N_{k-1} \geq 0 \\ N_{1}+\cdots+N_{k-1}=m}} \mathbb{F}_{N_{1}, \ldots, N_{k-1} ; i}(n) .
$$


This leads to

$$
\sum_{m, n \geq 0} F_{k, i}(m, n) x^{m} q^{n}=\sum_{N_{1} \geq N_{2} \geq \ldots \geq N_{k-1} \geq 0} x^{N_{1}+\cdots+N_{k-1}} \sum_{\lambda \in \mathbb{F}_{N_{1}, \ldots, N_{k-1} ; i}} q^{|\lambda|} .
$$

Hence Theorem 3.4 immediately follows when we show that for $k \geq i \geq 1$ and $N_{1} \geq$ $N_{2} \geq \cdots \geq N_{k-1} \geq 0$,

$$
\begin{aligned}
& \sum_{\lambda \in \mathbb{F}_{N_{1}, \ldots, N_{k-1} ; i}} q^{|\lambda|} \\
& =\frac{\left(-q^{2-2 N_{1}} ; q^{2}\right)_{N_{1}-1}\left(-q^{1-2 N_{1}} ; q^{2}\right)_{N_{1}} q^{2\left(N_{1}^{2}+\cdots+N_{k-1}^{2}+N_{i}+\cdots+N_{k-1}\right)}}{\left(q^{2} ; q^{2}\right)_{N_{1}-N_{2}} \cdots\left(q^{2} ; q^{2}\right)_{N_{k-2}-N_{k-1}}\left(q^{2} ; q^{2}\right)_{N_{k-1}}} .
\end{aligned}
$$

It turns out that the proof of (3.11) is more complicated than the proof of (3.1) due to Kurşungöz. To prove (3.11), we require to build two bijections. More precisely, let $\mathbb{G}_{N_{1}, \ldots, N_{k-1} ; i}(n)$ denote the set of overpartitions in $\mathbb{F}_{N_{1}, \ldots, N_{k-1} ;}(n)$ for which there are no overlined even parts and non-overlined odd parts. Let $\mathbb{E}_{N_{1}, \ldots, N_{k-1} ;}(n)$ denote the set of overpartitions in $\mathbb{G}_{N_{1}, \ldots, N_{k-1} ; i}(n)$ for which there are no overlined odd parts.

Set

$$
\mathbb{G}_{N_{1}, \ldots, N_{k-1} ; i}=\bigcup_{n \geq 0} \mathbb{G}_{N_{1}, \ldots, N_{k-1} ; i}(n),
$$

and

$$
\mathbb{E}_{N_{1}, \ldots, N_{k-1} ; i}=\bigcup_{n \geq 0} \mathbb{E}_{N_{1}, \ldots, N_{k-1} ; i}(n) .
$$

We will give bijective proofs of the following two relations in Section 4 and Section 5 respectively.

Lemma 3.5. For $k \geq i \geq 1$ and $N_{1} \geq N_{2} \geq \cdots \geq N_{k-1} \geq 0$,

$$
\sum_{\lambda \in \mathbb{F}_{N_{1}, \ldots, N_{k-1} ; i}} q^{|\lambda|}=\left(-q^{2-2 N_{1}} ; q^{2}\right)_{N_{1}-1} \sum_{\mu \in \mathbb{G}_{N_{1}, \ldots, N_{k-1} ; i}} q^{|\mu|} .
$$

Lemma 3.6. For $k \geq i \geq 1$ and $N_{1} \geq N_{2} \geq \cdots \geq N_{k-1} \geq 0$,

$$
\sum_{\mu \in \mathbb{G}_{N_{1}, \ldots, N_{k-1} ; i}} q^{|\mu|}=\left(-q^{1-2 N_{1}} ; q^{2}\right)_{N_{1}} \sum_{\nu \in \mathbb{E}_{N_{1}, \ldots, N_{k-1} ; i}} q^{|\nu|} .
$$

In Section 6, we first give a proof of Theorem 3.4 by using Lemma 3.5 and Lemma 3.6, as well as (3.1) due to Kurşungöz. Then we complete the proof of Theorem 1.9 by using Theorem [3.4, together with Lemma 3.3 and the relation (3.6). In the remaining part of this paper, we mark parts of an overpartition in Göllnitz-Gordon marking. 


\section{Proof of Lemma 3.5}

Let $\mathbb{R}_{N}$ denote the set of partitions $\tau=\left(\tau_{1}, \tau_{2}, \ldots, \tau_{\ell}\right)$ with distinct negative even parts which lay in $[-2 N,-2]$, that is, $\tau_{j}$ is negative and even for $1 \leq j \leq \ell$ and $-2 N \leq \tau_{1}<$ $\tau_{2}<\cdots<\tau_{\ell} \leq-2$. It is easy to see that the generating function of partitions in $\mathbb{R}_{N}$ :

$$
\sum_{\tau \in \mathbb{R}_{N}} q^{|\tau|}=\left(1+q^{-2 N}\right)\left(1+q^{2-2 N}\right) \cdots\left(1+q^{-2}\right)=\left(-q^{-2 N} ; q^{2}\right)_{N}
$$

Thus, Lemma 3.5 is equivalent to the following combinatorial statement.

Theorem 4.1. For $k \geq i \geq 1$ and $N_{1} \geq N_{2} \geq \cdots \geq N_{k-1} \geq 0$, there is a bijection $\Phi$ between $\mathbb{F}_{N_{1}, \ldots, N_{k-1} ; i}$ and $\mathbb{R}_{N_{1}-1} \times \mathbb{G}_{N_{1}, \ldots, N_{k-1} ; i}$ such that for $\lambda \in \mathbb{F}_{N_{1}, \ldots, N_{k-1} ; i}$ and $\Phi(\lambda)=$ $(\tau, \mu) \in \mathbb{R}_{N_{1}-1} \times \mathbb{G}_{N_{1}, \ldots, N_{k-1} ; i}$, we have $|\lambda|=|\tau|+|\mu|$.

Observe that $\mathbb{G}_{N_{1}, \ldots, N_{k-1} ; i}$ is the set of overpartitions in $\mathbb{F}_{N_{1}, \ldots, N_{k-1} ; i}$ for which there are no overlined even parts and non-overlined odd parts, so the key point in the construction of the bijection $\Phi$ is to remove overlined even parts and non-overlined odd parts from an overpartition in $\mathbb{F}_{N_{1}, \ldots, N_{k-1} ; i}$ to obtain a new overpartition in $\mathbb{G}_{N_{1}, \ldots, N_{k-1} ; i}$. To this end, we will first define three subsets $\mathbb{F}_{N_{1}, \ldots, N_{k-1} ; i, p}, \overline{\mathbb{F}}_{N_{1}, \ldots, N_{k-1} ; i, p}$ and $\overrightarrow{\mathbb{F}}_{N_{1}, \ldots, N_{k-1} ; i, p}$ of $\mathbb{F}_{N_{1}, \ldots, N_{k-1} ; i}$. Then we build a bijection $\Phi_{p}$ between $\mathbb{F}_{N_{1}, \ldots, N_{k-1} ; i, p}$ and $\overline{\mathbb{F}}_{N_{1}, \ldots, N_{k-1} ; i, p}$ and a bijection $\Phi_{(p)}$ between $\mathbb{F}_{N_{1}, \ldots, N_{k-1} ; i, p}$ and $\overrightarrow{\mathbb{F}}_{N_{1}, \ldots, N_{k-1} ; i, p}$. It turns out that $\Phi_{(p)}$ can be obtained by iteratively using the bijection $\Phi_{p}$ and plays a crucial role in the construction of the bijection $\Phi$ in Theorem 4.1 .

Let $\lambda$ be an overpartition in $\mathbb{F}_{N_{1}, \ldots, N_{k-1} ; i}$. For $1 \leq r \leq k-1$, define $\lambda^{(r)}=\left(\lambda_{1}^{(r)}, \lambda_{2}^{(r)}, \ldots\right.$, $\left.\lambda_{N_{r}}^{(r)}\right)$ to be the $r$-th sub-overpartition of $\lambda$ whose parts are $r$-marked parts in the GöllnitzGordon marking of $\lambda$, where $\lambda_{1}^{(r)} \leq \lambda_{2}^{(r)} \leq \cdots \leq \lambda_{N_{r}}^{(r)}$. The $\lambda_{j}^{(r)}$ are called the $j$-th part of the $r$-th sub-overpartition $\lambda^{(r)}$ of $\lambda$.

Let $k \geq i \geq 1$ and $N_{1} \geq N_{2} \geq \cdots \geq N_{k-1} \geq 0$ be given. For $1<p \leq N_{1}$, the subsets $\mathbb{F}_{N_{1}, \ldots, N_{k-1} ; i, p}, \overline{\mathbb{F}}_{N_{1}, \ldots, N_{k-1} ; i, p}$ and $\overrightarrow{\mathbb{F}}_{N_{1}, \ldots, N_{k-1} ; i, p}$ are described by using the first sub-overpartition $\lambda^{(1)}=\left(\lambda_{1}^{(1)}, \lambda_{2}^{(1)}, \ldots, \lambda_{N_{1}}^{(1)}\right)$ of an overpartition $\lambda$ in $\mathbb{F}_{N_{1}, \ldots, N_{k-1} ; i}$, where $\lambda_{1}^{(1)} \leq \lambda_{2}^{(1)} \leq \cdots \leq \lambda_{N_{1}}^{(1)}$

$\diamond \mathbb{F}_{N_{1}, \ldots, N_{k-1} ; i, p}$ is the set of overpartitions $\lambda$ in $\mathbb{F}_{N_{1}, \ldots, N_{k-1} ; i}$ such that (1) $\lambda_{p}^{(1)}$ is a non-overlined odd part or an overlined even part; $(2) \lambda_{j}^{(1)}$ is an overlined odd part or a non-overlined even part, where $p+1 \leq j \leq N_{1}$.

$\diamond \overline{\mathbb{F}}_{N_{1}, \ldots, N_{k-1} ; i, p}$ is the set of overpartitions $\lambda$ in $\mathbb{F}_{N_{1}, \ldots, N_{k-1} ; i}$ such that (1) $\lambda_{p}^{(1)}$ is an overlined odd part or a non-overlined even part; (2) $\lambda_{p+1}^{(1)}$ is a non-overlined odd part or an overlined even part; $(3) \lambda_{j}^{(1)}$ is an overlined odd part or a non-overlined even part, where $p+2 \leq j \leq N_{1}$.

$\diamond \overrightarrow{\mathbb{F}}_{N_{1}, \ldots, N_{k-1} ; i, p}$ is the set of overpartitions $\lambda$ in $\mathbb{F}_{N_{1}, \ldots, N_{k-1} ; i}$ such that $\lambda_{j}^{(1)}$ is an overlined odd part or a non-overlined even part, where $p \leq j \leq N_{1}$. 
By definition, it is easy to see that for $1 \leq p \leq N_{1}-2$,

$$
\overline{\mathbb{F}}_{N_{1}, \ldots, N_{k-1} ; i, p} \subseteq \mathbb{F}_{N_{1}, \ldots, N_{k-1} ; i, p+1} \subseteq \overrightarrow{\mathbb{F}}_{N_{1}, \ldots, N_{k-1} ; i, p+2} .
$$

We are ready to present the bijection $\Phi_{p}$ between $\mathbb{F}_{N_{1}, \ldots, N_{k-1} ; i, p}$ and $\overline{\mathbb{F}}_{N_{1}, \ldots, N_{k-1} ; i, p}$ and the bijection $\Phi_{(p)}$ between $\mathbb{F}_{N_{1}, \ldots, N_{k-1} ; i, p}$ and $\overrightarrow{\mathbb{F}}_{N_{1}, \ldots, N_{k-1} ; i, p}$. The following lemma gives the bijection $\Phi_{p}$, which will be proved at the end of this section.

Lemma 4.2. For $1<p \leq N_{1}$, there is a bijection $\Phi_{p}$ between $\mathbb{F}_{N_{1}, \ldots, N_{k-1} ; i, p}$ and $\overline{\mathbb{F}}_{N_{1}, \ldots, N_{k-1} ; i, p}$. Furthermore, for $\lambda \in \mathbb{F}_{N_{1}, \ldots, N_{k-1} ; i, p}$ and $\mu=\Phi_{p}(\lambda) \in \overline{\mathbb{F}}_{N_{1}, \ldots, N_{k-1} ; i, p}$, we have

$$
|\mu|=|\lambda|+2, \quad \text { and } \quad \lambda_{j}^{(1)}=\mu_{j}^{(1)} \quad \text { for } j \neq p, p+1,
$$

where $\lambda_{j}^{(1)}\left(\right.$ resp. $\left.\mu_{j}^{(1)}\right)$ is the $j$-th part of the first sub-overpartition of $\lambda($ resp. $\mu)$.

Taking the above bijection $\Phi_{p}$ in successive to give the following lemma.

Lemma 4.3. For $1<p \leq N_{1}$, there is a bijection $\Phi_{(p)}$ between $\mathbb{F}_{N_{1}, \ldots, N_{k-1} ; i, p}$ and $\overrightarrow{\mathbb{F}}_{N_{1}, \ldots, N_{k-1} ; i, p}$. Furthermore, for $\lambda \in \mathbb{F}_{N_{1}, \ldots, N_{k-1} ; i, p}$ and $\mu=\Phi_{p}(\lambda) \in \overrightarrow{\mathbb{F}}_{N_{1}, \ldots, N_{k-1} ; i, p}$, we have

$$
|\mu|=|\lambda|+2 N_{1}-2 p+2, \quad \text { and } \quad \lambda_{j}^{(1)}=\mu_{j}^{(1)} \quad \text { for } j<p .
$$

Proof. Define $\Phi_{(p)}=\Phi_{N_{1}} \Phi_{N_{1}-1} \cdots \Phi_{p}$, by Lemma 4.2, it is easy to verify that $\Phi_{(p)}$ is a bijection between $\mathbb{F}_{N_{1}, \ldots, N_{k-1} ; i, p}$ and $\overrightarrow{\mathbb{F}}_{N_{1}, \ldots, N_{k-1} ; i, p}$ as desired.

Before giving a proof of Lemma 4.2, we give a proof of Theorem 4.1 by successively using the bijection $\Phi_{(p)}$ in Lemma 4.3.

Proof of Theorem 4.1. Let $\lambda$ be an overpartition in $\mathbb{F}_{N_{1}, \ldots, N_{k-1} ; i}$. We aim to define $\Phi(\lambda)=(\tau, \mu)$ such that $\tau$ is a partition in $\mathbb{R}_{N_{1}-1}$ and $\mu$ is an overpartition in $\mathbb{G}_{N_{1}, \ldots, N_{k-1} ; i}$ satisfying $|\lambda|=|\tau|+|\mu|$. We consider two cases.

Case 1. If there are no overlined even parts and non-overlined odd parts in $\lambda$, then set $\mu=\lambda$ and $\tau=\emptyset$. It is easy to see that $\mu \in \mathbb{G}_{N_{1}, \ldots, N_{k-1} ; i}$ and $|\lambda|=|\mu|$.

Case 2. If there are $s \geq 1$ overlined even parts or non-overlined odd parts in $\lambda$, then by the definition of Göllnitz-Gordon marking, these parts are marked with 1. If we assume that $\lambda^{(1)}=\left(\lambda_{1}^{(1)}, \lambda_{2}^{(1)}, \ldots, \lambda_{N_{1}}^{(1)}\right)$ is the first sub-overpartition of $\lambda$, then there are $s$ overlined even parts or non-overlined odd parts in $\lambda^{(1)}$, which are $\lambda_{j_{1}}^{(1)}, \lambda_{j_{2}}^{(1)}, \ldots, \lambda_{j_{s-1}}^{(1)}$ and $\lambda_{j_{s}}^{(1)}$, where $1 \leq j_{1}<j_{2}<\cdots<j_{s} \leq N_{1}$. Under this assumption, we see that $\lambda \in \mathbb{F}_{N_{1}, \ldots, N_{k-1} ; i, j_{s}}$. Note that the smallest part of $\lambda$ is an overlined odd part or a non-overlined even part, so $j_{1}>1$. Set

$$
\tau=\left(-2\left(N_{1}-j_{1}+1\right),-2\left(N_{1}-j_{2}+1\right), \ldots,-2\left(N_{1}-j_{s}+1\right)\right),
$$


obviously, $\tau$ is a partition in $\mathbb{R}_{N_{1}-1}$. The partition $\mu$ can be obtained from $\lambda$ by employing the bijection in Lemma $4.3 s$ times. We denote the intermediate partitions by $\gamma^{0}, \gamma^{1}, \ldots, \gamma^{s}$ with $\gamma^{0}=\lambda$ and $\gamma^{s}=\mu$. For $1 \leq b \leq s$, the intermediate partition $\gamma^{b}$ can be obtained from $\gamma^{b-1}$ by using $\Phi_{\left(j_{s-b+1}\right)}$ in Lemma 4.3, that is, for $1 \leq b \leq s$,

$$
\gamma^{b}=\Phi_{\left(j_{s-b+1}\right)}\left(\gamma^{b-1}\right) \text {. }
$$

Note that $\gamma^{0} \in \mathbb{F}_{N_{1}, \ldots, N_{k-1} ; i, j_{s}}$, so by Lemma 4.3, we see that

$$
\gamma^{1} \in \mathbb{F}_{N_{1}, \ldots, N_{k-1} ; i, j_{s-1}} \text { and }\left|\gamma^{1}\right|=|\lambda|+2\left(N_{1}-j_{s}+1\right),
$$

and the first $\left(j_{s}-1\right)$ 1-marked parts in the Göllnitz-Gordon marking of $\gamma^{1}$ and $\gamma^{0}=\lambda$ are the same.

Employing Lemma 4.3 repeatedly, we derive that for $1 \leq b \leq s-1$,

$$
\gamma^{b} \in \mathbb{F}_{N_{1}, \ldots, N_{k-1} ; i, j_{s-b}} \text { and }\left|\gamma^{b}\right|=|\lambda|+2 \sum_{r=1}^{b}\left(N_{1}-j_{s-r+1}+1\right),
$$

and

$$
\gamma^{s} \in \overrightarrow{\mathbb{F}}_{N_{1}, \ldots, N_{k-1} ; i, j_{1}} \text { and }\left|\gamma^{s}\right|=|\lambda|+2 \sum_{r=1}^{s}\left(N_{1}-j_{s-r+1}+1\right) .
$$

Furthermore, for $1 \leq b \leq s$, the first $\left(j_{s-b+1}-1\right)$ 1-marked parts in the Göllnitz-Gordon marking of $\gamma^{b}$ and $\gamma^{0}=\lambda$ are the same. From the preceding assumption, we see that the first $\left(j_{1}-1\right)$ parts in the first sub-overpartition of $\lambda$ are non-overlined even parts or overlined odd parts, and so we derive that there are no overlined even parts or nonoverlined odd parts in $\gamma^{s}$. Hence

$$
\mu=\gamma^{s} \in \mathbb{G}_{N_{1}, \ldots, N_{k-1} ; i} \text { and }|\mu|=|\lambda|+2 \sum_{r=1}^{s}\left(N_{1}-j_{s-r+1}+1\right),
$$

and it is easy to check that $|\tau|+|\mu|=|\lambda|$. Therefore $\Phi$ is well-defined.

To prove that $\Phi$ is a bijection, we shall give a brief description of the inverse map $\Psi$ of $\Phi$. Let $\mu$ be an overpartition in $\mathbb{G}_{N_{1}, \ldots, N_{k-1} ; i}$ and $\tau$ be a partition with distinct negative even parts lying in $\left[2-2 N_{1},-2\right]$. We shall define $\Psi(\tau, \mu)=\lambda$ such that $\lambda$ is an overpartition in $\mathbb{F}_{N_{1}, \ldots, N_{k-1} ; i}$ and $|\lambda|=|\tau|+|\mu|$. There are two cases.

Case 1. $\tau=\emptyset$. In this event, set $\lambda=\mu$. Note that $\mathbb{G}_{N_{1}, \ldots, N_{k-1} ; i} \subseteq \mathbb{F}_{N_{1}, \ldots, N_{k-1} ; i}$, so $\lambda \in \mathbb{F}_{N_{1}, \ldots, N_{k-1} ; i}$ and there are no overlined even parts and non-overlined odd parts in $\lambda$.

Case 2. $\tau \neq \emptyset$. In this event, assume that

$$
\tau=\left(-2\left(N_{1}-j_{1}+1\right),-2\left(N_{1}-j_{2}+1\right), \ldots,-2\left(N_{1}-j_{s}+1\right)\right),
$$

where $1<j_{1}<j_{2}<\cdots<j_{s} \leq N_{1}$. The partition $\lambda$ can be recovered from $\mu$ by using the bijection in Lemma $4.3 s$ times. We denote the intermediate partitions by $\delta^{0}, \delta^{1}, \ldots, \delta^{s}$ 
with $\delta^{s}=\mu$ and $\delta^{0}=\lambda$. For $1 \leq b \leq s$, the intermediate partition $\delta^{b-1}$ can be obtained from $\delta^{b}$ by using the bijection $\Phi_{\left(j_{s-b+1}\right)}^{-1}$, that is $\delta^{b-1}=\Phi_{\left(j_{s-b+1}\right)}^{-1}\left(\delta^{b}\right)$. Set $\lambda=\delta^{0}$, it follows from Lemma 4.3 that $\lambda$ is an overpartition in $\mathbb{F}_{N_{1}, \ldots, N_{k-1} ; i}$ and $|\lambda|=|\tau|+|\mu|$, and $\Psi(\Phi(\lambda))=\lambda$ for any $\lambda$ in $\mathbb{F}_{N_{1}, \ldots, N_{k-1} ; i}$. Hence $\Phi$ is a bijection between $\mathbb{F}_{N_{1}, \ldots, N_{k-1} ; i}$ and $\mathbb{R}_{N_{1}-1} \times \mathbb{G}_{N_{1}, \ldots, N_{k-1} ; i}$. This completes the proof of Theorem 4.1, and hence Lemma 3.5] is verified.

It remains to show Lemma 4.2. To this end, we shall divide $\mathbb{F}_{N_{1}, \ldots, N_{k-1} ; i, p}$ into four disjoint subsets $\mathbb{F}_{N_{1}, \ldots, N_{k-1} ; i, p}^{(l)}(1 \leq l \leq 4)$ and divide $\overline{\mathbb{F}}_{N_{1}, \ldots, N_{k-1} ; i, p}$ into four disjoint subsets $\overline{\mathbb{F}}_{N_{1}, \ldots, N_{k-1} ; i, p}^{(l)}(1 \leq l \leq 4)$. We then construct the bijection $\Phi_{p}$ consisting of four bijections $\Phi_{l, p}$ between $\mathbb{F}_{N_{1}, \ldots, N_{k-1} ; i, p}^{(l)}$ and $\overline{\mathbb{F}}_{N_{1}, \ldots, N_{k-1} ; i, p}^{(l)}$, where $1 \leq l \leq 4$.

For $1<p \leq N_{1}$, let $\lambda^{(1)}=\left(\lambda_{1}^{(1)}, \lambda_{2}^{(1)}, \ldots, \lambda_{N_{1}}^{(1)}\right)$ be the first sub-overpartition of $\lambda$ in $\mathbb{F}_{N_{1}, \ldots, N_{k-1} ; i, p}$, by definition, we see that $\lambda_{p}^{(1)}$ is a non-overlined odd part or an overlined even part and $\lambda_{j}^{(1)}$ is an overlined odd part or a non-overlined even part, where $p+1 \leq j \leq N_{1}$. The subsets $\mathbb{F}_{N_{1}, \ldots, N_{k-1} ; i, p}^{(l)}$ can be described in terms of the first sub-overpartition of $\lambda$.

(1) $\mathbb{F}_{N_{1}, \ldots, N_{k-1} ; i, p}^{(1)}$ is the set of overpartitions $\lambda$ in $\mathbb{F}_{N_{1}, \ldots, N_{k-1} ; i, p}$ such that (i) $\lambda_{p}^{(1)}$ is a non-overlined odd part; (ii) there are no non-overlined even parts of size $\left|\lambda_{p}^{(1)}\right|+1$ in $\lambda$ when $\left|\lambda_{p-1}^{(1)}\right| \leq\left|\lambda_{p}^{(1)}\right|-2$.

(2) $\mathbb{F}_{N_{1}, \ldots, N_{k-1} ; i, p}^{(2)}$ is the set of overpartitions $\lambda$ in $\mathbb{F}_{N_{1}, \ldots, N_{k-1} ; i, p}$ such that (i) $\lambda_{p}^{(1)}$ is a nonoverlined odd part; (ii) there is at least one non-overlined even part of size $\left|\lambda_{p}^{(1)}\right|+1$ in $\lambda$; (iii) $\left|\lambda_{p-1}^{(1)}\right| \leq\left|\lambda_{p}^{(1)}\right|-2$.

(3) $\mathbb{F}_{N_{1}, \ldots, N_{k-1} ; i, p}^{(3)}$ is the set of overpartitions $\lambda$ in $\mathbb{F}_{N_{1}, \ldots, N_{k-1} ; i, p}$ such that (i) $\lambda_{p}^{(1)}$ is an overlined even part; (ii) there is no overlined odd part of size $\left|\lambda_{p}^{(1)}\right|+1$ in $\lambda$.

(4) $\mathbb{F}_{N_{1}, \ldots, N_{k-1} ; i, p}^{(4)}$ is the set of overpartitions $\lambda$ in $\mathbb{F}_{N_{1}, \ldots, N_{k-1} ; i, p}$ such that (i) $\lambda_{p}^{(1)}$ is an overlined even part; (ii) there is an overlined odd part of size $\left|\lambda_{p}^{(1)}\right|+1$ in $\lambda$.

For $1<p \leq N_{1}$, let $\mu^{(1)}=\left(\mu_{1}^{(1)}, \mu_{2}^{(1)}, \ldots, \mu_{N_{1}}^{(1)}\right)$ be the first sub-overpartition of $\mu$ in $\overline{\mathbb{F}}_{N_{1}, \ldots, N_{k-1} ; i, p}$, by definition, we see that $\mu_{p}^{(1)}$ is an overlined odd part or a non-overlined even part, $\mu_{p+1}^{(1)}$ is a non-overlined odd part or an overlined even part, and $\mu_{j}^{(1)}$ is an overlined odd part or a non-overlined even part, where $p+2 \leq j \leq N_{1}$. We shall divide the overpartitions $\mu$ in $\overline{\mathbb{F}}_{N_{1}, \ldots, N_{k-1} ; i, p}$ into four disjoint subsets $\overline{\mathbb{F}}_{N_{1}, \ldots, N_{k-1} ; i, p}^{(l)}$, where $1 \leq l \leq 4$ in terms of the first sub-overpartition of $\mu$. When $p \geq N_{1}$, we see that $\mu_{p+1}^{(1)}$ does not occur in $\mu$. For convenience, set $\left|\mu_{N_{1}+1}^{(1)}\right|=\infty$. 
(1) $\overline{\mathbb{F}}_{N_{1}, \ldots, N_{k-1} ; i, p}^{(1)}$ is the set of overpartitions $\mu$ in $\overline{\mathbb{F}}_{N_{1}, \ldots, N_{k-1} ; i, p}$ such that (i) $\mu_{p}^{(1)}$ is an overlined odd part; (ii) there are no non-overlined even parts of size $\left|\mu_{p}^{(1)}\right|+1$ in $\mu$ when $\left|\mu_{p+1}^{(1)}\right| \geq\left|\mu_{p}^{(1)}\right|+2$.

(2) $\overline{\mathbb{F}}_{N_{1}, \ldots, N_{k-1} ; i, p}^{(2)}$ is the set of overpartitions $\mu$ in $\overline{\mathbb{F}}_{N_{1}, \ldots, N_{k-1} ; i, p}$ such that (i) $\mu_{p}^{(1)}$ is a non-overlined even part; (ii) there is an overlined odd part of size $\left|\mu_{p}^{(1)}\right|+1$ in $\mu$; (iii) there are no non-overlined even parts of size $\left|\mu_{p}^{(1)}\right|+2$ in $\mu$ when $\left|\mu_{p+1}^{(1)}\right|>\left|\mu_{p}^{(1)}\right|+2$.

(3) $\overline{\mathbb{F}}_{N_{1}, \ldots, N_{k-1} ; i, p}^{(3)}$ is the set of overpartitions $\mu$ in $\overline{\mathbb{F}}_{N_{1}, \ldots, N_{k-1} ; i, p}$ such that (i) $\mu_{p}^{(1)}$ is a non-overlined even part; (ii) there is no overlined odd part of size $\left|\mu_{p}^{(1)}\right|+1$ in $\mu$.

(4) $\overline{\mathbb{F}}_{N_{1}, \ldots, N_{k-1} ; i, p}^{(4)}$ is the set of overpartitions $\mu$ in $\overline{\mathbb{F}}_{N_{1}, \ldots, N_{k-1} ; i, p}$ such that (i) if $\mu_{p}^{(1)}$ is an overlined odd part, then there is at least one non-overlined even part of size $\left|\mu_{p}^{(1)}\right|+1$ in $\mu$, and $\left|\mu_{p+1}^{(1)}\right| \geq\left|\mu_{p}^{(1)}\right|+2$; (ii) if $\mu_{p}^{(1)}$ is a non-overlined even part, then there are an overlined odd part of size $\left|\mu_{p}^{(1)}\right|+1$ and at least one non-overlined even part of size $\left|\mu_{p}^{(1)}\right|+2$ in $\mu$, and $\left|\mu_{p+1}^{(1)}\right|>\left|\mu_{p}^{(1)}\right|+2$.

We are now ready to define the bijections $\Phi_{l, p}$ between $\mathbb{F}_{N_{1}, \ldots, N_{k-1} ; i, p}^{(l)}$ and $\overline{\mathbb{F}}_{N_{1}, \ldots, N_{k-1} ; i, p}^{(l)}$, where $1 \leq l \leq 4$.

Lemma 4.4. For $1<p \leq N_{1}$, there is a bijection $\Phi_{1, p}$ between $\mathbb{F}_{N_{1}, \ldots, N_{k-1} ; i, p}^{(1)}$ and $\overline{\mathbb{F}}_{N_{1}, \ldots, N_{k-1} ; i, p}^{(1)}$. Furthermore, for $\lambda \in \mathbb{F}_{N_{1}, \ldots, N_{k-1} ; i, p}^{(1)}$ and $\mu=\Phi_{1, p}(\lambda) \in \overline{\mathbb{F}}_{N_{1}, \ldots, N_{k-1} ; i, p}^{(1)}$, we have

$$
|\mu|=|\lambda|+2, \quad \text { and } \quad \lambda_{j}^{(1)}=\mu_{j}^{(1)} \quad \text { for } j \neq p, p+1 .
$$

Proof. Let $\lambda^{(1)}=\left(\lambda_{1}^{(1)}, \lambda_{2}^{(1)}, \ldots, \lambda_{N_{1}}^{(1)}\right)$ be the first sub-overpartition of $\lambda$ in $\mathbb{F}_{N_{1}, \ldots, N_{k-1} ; i, p}^{(1)}$. By definition, we see that $\lambda_{p}^{(1)}$ is a non-overlined odd part, set $\lambda_{p}^{(1)}=2 t+1$. Since $\lambda \in \mathbb{F}_{N_{1}, \ldots, N_{k-1} ; i, p}^{(1)}$, we see that $2 t+2$ does not occur in $\lambda$ when $\left|\lambda_{p-1}^{(1)}\right| \leq 2 t-1$ and $\lambda_{j}^{(1)}$ is an overlined odd part or a non-overlined even part, where $p+1 \leq j \leq N_{1}$. When $1<p<N_{1}$, set $\lambda_{p+1}^{(1)}=\overline{2 a+1}$ or $2 a+2$, it follows from the definition of Göllnitz-Gordon marking that $a \geq t+1$.

For $1<p \leq N_{1}$, define $\mu=\Phi_{1, p}(\lambda)$, which can be obtained from $\lambda$ by performing the following two operations.

(1) Replace $\lambda_{p}^{(1)}=2 t+1$ by $\overline{2 t+3}$.

(2) When $1<p<N_{1}$, replace $\lambda_{p+1}^{(1)}$ by $2 a+1$ (or $\overline{2 a+2}$ ) if $\lambda_{p+1}^{(1)}=\overline{2 a+1}$ (or $2 a+2$ ); When $p=N_{1}$, we shall do nothing.

Obviously, $|\mu|=|\lambda|+2$. We first prove that the parts from $\lambda$ in $\mu$ have the same marks as in $\lambda$ and the new generated parts $\overline{2 t+3}$ and $2 a+1$ (or $\overline{2 a+2}$ ) in $\mu$ are marked with 1. This leads to $\mu \in \mathbb{F}_{N_{1}, \ldots, N_{k-1} ; i}$. By the definition of $\mu$, it is obvious that this assertion 
is true for the parts of size not exceed $2 t+1$ in $\mu$. We next show that the parts $2 t+2$ in $\mu$ have the same marks as in $\lambda$ and the new generated part $\overline{2 t+3}$ in $\mu$ is marked with 1. There are two cases: if $\lambda_{p-1}^{(1)}=\overline{2 t}$, or $2 t$, or $\overline{2 t+1}$, or $2 t+1$, then $\mu_{p-1}^{(1)}=\overline{2 t}$, or $2 t$, or $\overline{2 t+1}$, or $2 t+1$, it follows from the definition of Göllnitz-Gordon marking that the parts $2 t+2$ in $\mu$ have the same marks as in $\lambda$ even if the part $\lambda_{p}^{(1)}=2 t+1$ replaced by $\overline{2 t+3}$ in $\mu$. Furthermore, the new generated part $\overline{2 t+3}$ in $\mu$ is marked with 1 since there is no 1-marked part of size $2 t+2$ in $\mu$. If $\left|\lambda_{p-1}^{(1)}\right| \leq 2 t-1$, then $2 t+2$ does not occur in $\lambda$, and so neither in $\mu$. It follows that the new generated part $\overline{2 t+3}$ in $\mu$ is marked with 1. Therefore, in either case, the parts $2 t+2$ in $\mu$ have the same marks as in $\lambda$ and the new generated part $\overline{2 t+3}$ in $\mu$ is marked with 1 . By the definition of Göllnitz-Gordon marking, it is easy to see that the new generated part $2 a+1$ (or $\overline{2 a+2}$ ) in $\mu$ replacing $\lambda_{p+1}^{(1)}$ is marked with 1 , which has the same size with $\lambda_{p+1}^{(1)}$ in $\lambda$, and so the parts of size larger than $2 t+2$ in $\mu$ have the same marks as in $\lambda$. Thus, we arrive at our assertion and prove that $\mu \in \mathbb{F}_{N_{1}, \ldots, N_{k-1} ; i}$.

Let $\mu^{(1)}=\left(\mu_{1}^{(1)}, \mu_{2}^{(1)}, \ldots, \mu_{N_{1}}^{(1)}\right)$ be the first sub-overpartition of $\mu$. It follows from the above proof that $\lambda_{j}^{(1)}=\mu_{j}^{(1)}$ for $j \neq p, p+1$. Furthermore, $\mu_{p}^{(1)}=\overline{2 t+3}, \mu_{p+1}^{(1)}$ is a non-overlined odd part or an overlined even part, and $\mu_{j}^{(1)}$ is an overlined odd part or a non-overlined even part, where $p+2 \leq j \leq N_{1}$. Moreover, if $\left|\mu_{p+1}^{(1)}\right| \geq 2 t+5$, then $2 t+4$ does not occur in $\mu$. This is because that $2 t+4$ does not occur in $\lambda$ when $\left|\lambda_{p+1}^{(1)}\right| \geq 2 t+5$ and $\lambda_{p}^{(1)}=2 t+1$. Therefore, $\mu \in \overline{\mathbb{F}}_{N_{1}, \ldots, N_{k-1} ; i, p}^{(1)}$. Furthermore, it is not difficult to show that $\Phi_{1, p}$ is reversible. So, we conclude that $\Phi_{1, p}$ is a bijection between $\mathbb{F}_{N_{1}, \ldots, N_{k-1} ; i, p}^{(1)}$ and $\overline{\mathbb{F}}_{N_{1}, \ldots, N_{k-1} ; i, p}^{(1)}$. This completes the proof.

For example, for $p=3$, let

$$
G G(\lambda)=\left[\begin{array}{llllll} 
& & & & 6 & \\
& 2 & & 4 & & \overline{7} \\
\overline{1} & & 3^{2} & & \mathbf{6} &
\end{array}\right] \begin{aligned}
& 3 \\
& 2 \\
& 1
\end{aligned}
$$

be the Göllnitz-Gordon marking representation of $\lambda$ in $\mathbb{F}_{4,3,1 ; 3,3}^{(1)}$. Applying the bijection $\Phi_{1,3}$ to $\lambda$, we get

$$
G G(\mu)=\left[\begin{array}{lllllll} 
& & & & & 6 & \\
& 2 & & 4 & & & \overline{7} \\
\overline{1} & & 3 & & \overline{\mathbf{5}} & \overline{\mathbf{6}} &
\end{array}\right] \begin{aligned}
& 3 \\
& 2 \\
& 1
\end{aligned}
$$

which is in $\overline{\mathbb{F}}_{4,3,1 ; 3,3}^{(1)}$.

Lemma 4.5. For $1<p \leq N_{1}$, there is a bijection $\Phi_{2, p}$ between $\mathbb{F}_{N_{1}, \ldots, N_{k-1} ; i, p}^{(2)}$ and $\overline{\mathbb{F}}_{N_{1}, \ldots, N_{k-1} ; i, p}^{(2)}$. Furthermore, for $\lambda \in \mathbb{F}_{N_{1}, \ldots, N_{k-1} ; i, p}^{(2)}$ and $\mu=\Phi_{2, p}(\lambda) \in \overline{\mathbb{F}}_{N_{1}, \ldots, N_{k-1} ; i, p}^{(2)}$, we have

$$
|\mu|=|\lambda|+2, \quad \text { and } \quad \lambda_{j}^{(1)}=\mu_{j}^{(1)} \quad \text { for } j \neq p, p+1 .
$$


Proof. Let $\lambda^{(1)}=\left(\lambda_{1}^{(1)}, \lambda_{2}^{(1)}, \ldots, \lambda_{N_{1}}^{(1)}\right)$ be the first sub-overpartition of $\lambda$ in $\mathbb{F}_{N_{1}, \ldots, N_{k-1} ; i, p}^{(2)}$. By definition, we see that $\lambda_{p}^{(1)}$ is a non-overlined odd part, set $\lambda_{p}^{(1)}=2 t+1$. Note that $\lambda \in \mathbb{F}_{N_{1}, \ldots, N_{k-1} ; i, p}^{(2)}$, we see that $\left|\lambda_{p-1}^{(1)}\right| \leq 2 t-1$ and there is at least one part $2 t+2$ in $\lambda$. Furthermore, $\lambda_{p+1}^{(1)}$ is an overlined odd part or a non-overlined even part, set $\lambda_{p+1}^{(1)}=\overline{2 a+1}$ or $2 a+2$. From the definition of Göllnitz-Gordon marking, we see that $a \geq t+1$.

To define $\Phi_{2, p}$, we introduce an index $r$ which is related to the marks of the parts $2 t+2$ in $\lambda$. If there exists an integer $b$ such that there are $b$-marked parts $2 t$ and $2 t+2$ in $\lambda$, then set $r=b$; Otherwise, we define $r$ as the largest mark of the parts $2 t+2$ in $\lambda$. Since $2 t+2$ occurs in $\lambda$ and $\lambda_{p}^{(1)}=2 t+1$, by the definition of Göllnitz-Gordon marking, we deduce that the marks of the parts $2 t+2$ in $\lambda$ are larger than 1 . Hence $r \geq 2$.

For $1<p \leq N_{1}$, define $\mu=\Phi_{2, p}(\lambda)$, which can be obtained from $\lambda$ by doing the following two operations.

(1) Replace $\lambda_{p}^{(1)}=2 t+1$ by $2 t+2$ and replace the $r$-marked part $2 t+2$ in $\lambda$ by $\overline{2 t+3}$.

(2) When $1<p<N_{1}$, replace $\lambda_{p+1}^{(1)}$ by $2 a+1$ (or $\overline{2 a+2}$ ) if $\lambda_{p+1}^{(1)}=\overline{2 a+1}$ (or $2 a+2$ ); When $p=N_{1}$, we shall do nothing.

Obviously, $|\mu|=|\lambda|+2$. We first show that $\mu$ is an overpartition in $\mathbb{F}_{N_{1}, \ldots, N_{k-1} ; i}$. To this end, we shall show that the parts from $\lambda$ in $\mu$ have the same marks as in $\lambda$ and the new generated parts $2 t+2$ and $2 a+1$ (or $\overline{2 a+2}$ ) in $\mu$ are marked with 1 , and the new generated part $\overline{2 t+3}$ in $\mu$ is marked with $r$. By the definition of $\mu$, it is obvious that the marks of parts of size not exceed $2 t+1$ in $\mu$ are the same as in $\lambda$. Note that $\left|\lambda_{p-1}^{(1)}\right| \leq 2 t-1$, $\lambda_{p}^{(1)}=2 t+1$ and $\left|\lambda_{p+1}^{(1)}\right|>2 t+1$, this implies that there are no 1 -marked parts $\overline{2 t}, 2 t$ and $\overline{2 t+1}$ in $\lambda$, and there is only one part $2 t+1$ in $\lambda$, that is $\lambda_{p}^{(1)}$. Therefore there are no 1-marked parts $\overline{2 t}, 2 t, \overline{2 t+1}$ and $2 t+1$ in $\mu$. By the definition of Göllnitz-Gordon marking, we see that the new generated part $2 t+2$ in $\mu$ replacing $\lambda_{p}^{(1)}=2 t+1$ should be marked with 1 and the parts $2 t+2$ from $\lambda$ in $\mu$ have the same marks as in $\lambda$. We proceed to show that the new generated part $\overline{2 t+3}$ in $\mu$ replacing the $r$-marked part $2 t+2$ in $\lambda$ is also marked with $r$. By the definition of Göllnitz-Gordon marking, we see that there are $f$-marked $(2 t+2)$ 's in $\lambda$ where $2 \leq f \leq r-1$. It follows from the preceding proof that there are also $f$-marked $(2 t+2)$ 's in $\mu$, where $1 \leq f \leq r-1$. Therefore the new generated part $\overline{2 t+3}$ in $\mu$ replacing the $r$-marked $2 t+2$ in $\lambda$ is also marked with $r$. Again, from the definition of Göllnitz-Gordon marking, it is easy to see that the new generated part $2 a+1$ (or $\overline{2 a+2}$ ) in $\mu$ replacing $\lambda_{p+1}^{(1)}$ is marked with 1 , which has the same size with $\lambda_{p+1}^{(1)}$ in $\lambda$, and so the parts of size larger than $2 t+1$ in $\mu$ have the same marks as in $\lambda$. Thus, we prove that $\mu$ is an overpartition in $\mathbb{F}_{N_{1}, \ldots, N_{k-1} ; i}$.

Let $\mu^{(1)}=\left(\mu_{1}^{(1)}, \mu_{2}^{(1)}, \ldots, \mu_{N_{1}}^{(1)}\right)$ be the first sub-overpartition of $\mu$. From the above proof, we see that $\lambda_{j}^{(1)}=\mu_{j}^{(1)}$ for $j \neq p, p+1$. Furthermore $\mu_{p}^{(1)}=2 t+2, \mu_{p+1}^{(1)}$ is a non-overlined odd part or an overlined even part, and $\mu_{j}^{(1)}$ is an overlined odd part or a non-overlined even part, where $p+2 \leq j \leq N_{1}$. This proves that $\mu$ is an overpartition in $\overline{\mathbb{F}}_{N_{1}, \ldots, N_{k-1} ; i, p}$. Again by the preceding proof, we also see that there is an $r$-marked 
$\overline{2 t+3}$ in $\mu$. Furthermore, from the definition of $\lambda$, we see that $2 t+4$ does not occur in $\lambda$ if $\left|\lambda_{p+1}^{(1)}\right|>2 t+4$, otherwise, it contradicts to the assumptions that $\lambda_{p}^{(1)}=2 t+1$ and $\left|\lambda_{p+1}^{(1)}\right|>2 t+4$. Hence, we derive that $2 t+4$ does not occur in $\mu$ if $\left|\mu_{p+1}^{(1)}\right|>2 t+4$. This proves that $\mu$ is an overpartition in $\overline{\mathbb{F}}_{N_{1}, \ldots, N_{k-1} ; i, p}^{(2)}$. Moreover, it can be checked that $\Phi_{2, p}$ is reversible. So we conclude that $\Phi_{2, p}$ is a bijection between $\mathbb{F}_{N_{1}, \ldots, N_{k-1} ; i, p}^{(2)}$ and $\overline{\mathbb{F}}_{N_{1}, \ldots, N_{k-1} ; i, p}^{(2)}$. This completes the proof.

For example, for $p=3$, let

$$
G G(\lambda)=\left[\begin{array}{llllllll} 
& & 4 & & 8 & & & \\
& 2 & 4 & & 8 & & \overline{11} & \\
\overline{1} & & \overline{4} & 7 & & \mathbf{1 0} & & \overline{13}
\end{array}\right] \begin{aligned}
& 3 \\
& 2 \\
& 1
\end{aligned}
$$

be a Göllnitz-Gordon marking representation of $\lambda$ in $\mathbb{F}_{5,4,2 ; 3,3}^{(2)}$. Applying the bijection $\Phi_{2,3}$ to $\lambda$, we get

$$
G G(\mu)=\left[\begin{array}{llllllll} 
& & 4 & & \overline{\mathbf{9}} & & \\
& 2 & 4 & & 8 & & \overline{11} & \\
\overline{1} & & \overline{4} & & \mathbf{8} & \overline{\mathbf{1 0}} & & \overline{13}
\end{array}\right] \begin{aligned}
& 3 \\
& 2 \\
& 1
\end{aligned},
$$

which is in $\overline{\mathbb{F}}_{5,4,2 ; 3,3}^{(2)}$.

Lemma 4.6. For $1<p \leq N_{1}$, there is a bijection $\Phi_{3, p}$ between $\mathbb{F}_{N_{1}, \ldots, N_{k-1} ; i, p}^{(3)}$ and $\overline{\mathbb{F}}_{N_{1}, \ldots, N_{k-1} ; i, p}^{(3)}$. Furthermore, for $\lambda \in \mathbb{F}_{N_{1}, \ldots, N_{k-1} ; i, p}^{(3)}$ and $\mu=\Phi_{3, p}(\lambda) \in \overline{\mathbb{F}}_{N_{1}, \ldots, N_{k-1} ; i, p}^{(3)}$, we have

$$
|\mu|=|\lambda|+2, \quad \text { and } \quad \lambda_{j}^{(1)}=\mu_{j}^{(1)} \quad \text { for } j \neq p, p+1 .
$$

Proof. Let $\lambda^{(1)}=\left(\lambda_{1}^{(1)}, \lambda_{2}^{(1)}, \ldots, \lambda_{N_{1}}^{(1)}\right)$ be the first sub-overpartition of $\lambda$ in $\mathbb{F}_{N_{1}, \ldots, N_{k-1} ; i, p}^{(3)}$. By definition, we see that $\lambda_{p}^{(1)}$ is an overlined even part, set $\lambda_{p}^{(1)}=\overline{2 t}$. Note that $\lambda \epsilon$ $\mathbb{F}_{N_{1}, \ldots, N_{k-1} ; i, p}^{(3)}$, so $\overline{2 t+1}$ does not occur in $\lambda$ and $\lambda_{j}^{(1)}$ is an overlined odd part or a nonoverlined even part, where $p+1 \leq j \leq N_{1}$. This implies that there are no parts of size $2 t+1$ in $\lambda$. Set $\lambda_{p+1}^{(1)}=\overline{2 a+1}$ or $2 a+2$, where $a \geq t+1$.

To define $\Phi_{3, p}$, we introduce an index $r$ which is related to the marks of the parts $2 t$ in $\lambda$. There are three cases: If $\lambda_{p-1}^{(1)}=\overline{2 t-2}$, or $2 t-2$, or $\overline{2 t-1}$, or $2 t-1$, then set $r=1$; If $\left|\lambda_{p-1}^{(1)}\right| \leq 2 t-3$ and there exists an interger $b$ such that there are $b$-marked parts $2 t-2$ and $2 t$ in $\lambda$, then set $r=b$; Otherwise, set $r$ to be the largest mark of the parts of size $2 t$ in $\lambda$. Since $\lambda_{p}^{(1)}=\overline{2 t}$, we see that $r \geq 1$.

For $1<p \leq N_{1}$, define $\mu=\Phi_{3, p}(\lambda)$ which can be obtained from $\lambda$ by doing the following two operations:

(1) When $r=1$, replace $\lambda_{p}^{(1)}=\overline{2 t}$ by $2 t+2$; When $r \geq 2$, replace $\lambda_{p}^{(1)}=\overline{2 t}$ by $2 t$ and replace the $r$-marked part $2 t$ in $\lambda$ by $2 t+2$. 
(2) When $1<p<N_{1}$, replace $\lambda_{p+1}^{(1)}$ by $2 a+1$ (or $\overline{2 a+2}$ ) if $\lambda_{p+1}^{(1)}=\overline{2 a+1}($ or $2 a+2)$; When $p=N_{1}$, we shall do nothing.

Obviously, $|\mu|=|\lambda|+2$. We first show that $\mu$ is an overpartition in $\mathbb{F}_{N_{1}, \ldots, N_{k-1} ; i}$. We assert that the parts from $\lambda$ in $\mu$ have the same marks as in $\lambda$ and the new generated part $2 a+1$ (or $\overline{2 a+2}$ ) in $\mu$ is marked with 1 ; When $r \geq 1$, the new generated part $2 t+2$ in $\mu$ replacing the $r$-marked part of size $2 t$ is marked with $r$; When $r \geq 2$, the new generated part $2 t$ replacing $\lambda_{p}^{(1)}=\overline{2 t}$ is marked with 1 . From the construction of $\mu$ and the definition of Göllnitz-Gordon marking, it is obvious that the marks of parts of size not exceed $2 t-1$ in $\mu$ are the same as in $\lambda$. We proceed to show that this assertion holds for parts of size not exceed $2 t+2$ in $\mu$. We consider the following two cases:

(1) When $r=1$, there are two subcases:

(1.1) When $\lambda_{p-1}^{(1)}=\overline{2 t-2}$, or $2 t-2$, or $\overline{2 t-1}$, or $2 t-1$, by the definition of GöllnitzGordon marking, we see that the parts $2 t$ from $\lambda$ in $\mu$ have the same marks as in $\lambda$ and the new generated part $2 t+2$ in $\mu$ replacing $\lambda_{p}^{(1)}=\overline{2 t}$ should be marked with 1 . Since there are no parts of size $2 t+1$ in $\lambda$, from the construction of $\mu$, we see that there are no parts of size $2 t+1$ in $\mu$. Note that the new generated part $2 t+2$ in $\mu$ is marked with 1 , so we conclude that the marks of parts $2 t+2$ from $\lambda$ in $\mu$ are the same as in $\lambda$.

(1.2) When $\left|\lambda_{p-1}^{(1)}\right| \leq 2 t-3$, and there is only one part of size $2 t$ in $\lambda$, that is, $\lambda_{p}^{(1)}=\overline{2 t}$, from the construction of $\mu$, we see that there are no parts of size $2 t$ or $2 t+1$ in $\mu$ and the new generated part $2 t+2$ in $\mu$ replacing $\lambda_{p}^{(1)}=\overline{2 t}$ is marked with 1 . Furthermore, the marks of the parts $2 t+2$ from $\lambda$ in $\mu$ stay the same as in $\lambda$.

(2) When $r \geq 2$, then either there are $r$-marked parts $2 t-2$ and $2 t$ in $\lambda$, or $r$ is the largest mark of the parts $2 t$ in $\lambda$. By the definition of Göllnitz-Gordon marking, we see that there are $f$-marked parts of size $2 t$ in $\lambda$, where $1 \leq f \leq r$. It follows that the marks of the parts $2 t+2$ in $\lambda$ are greater than $r$. Note that $\left|\lambda_{p-1}^{(1)}\right| \leq 2 t-3$, so the new generated part $2 t$ in $\mu$ replacing $\lambda_{p}^{(1)}=\overline{2 t}$ is marked with 1 and the marks of parts $2 t$ from $\lambda$ in $\mu$ are the same as in $\lambda$. This means that there are $f$-marked $2 t$ 's in $\mu$, where $1 \leq f \leq r-1$. Since there are no parts of size $2 t+1$ in $\mu$, we deduce that the new generated part $2 t+2$ in $\mu$ replacing the $r$-marked part $2 t$ in $\lambda$ is marked with $r$ and the marks of the parts $2 t+2$ from $\lambda$ in $\mu$ are the same as in $\lambda$. Furthermore, $r$ is the smallest mark of the parts $2 t+2$ in $\mu$.

It remains to show that the assertion holds for parts of size greater than $2 t+2$ in $\mu$. By the definition of Göllnitz-Gordon marking, it is easy to see that the new generated $r$-marked part $2 t+2$ in $\mu$ replacing the $r$-marked part of size $2 t$ in $\lambda$ could affect the mark of the $r$-marked part of size $2 t+3$ or $2 t+4$ in $\mu$. Furthermore, the new generated part $2 a+1$ (or $\overline{2 a+2}$ ) in $\mu$ replacing $\lambda_{p+1}^{(1)}$ is marked with 1 , which has the same size with $\lambda_{p+1}^{(1)}$ in $\lambda$. Hence it suffices to show that the $r$-marked part of size $2 t+3$ or $2 t+4$ from $\lambda$ in $\mu$ is also marked with $r$ in $\mu$. We consider the following two cases:

(1) If $r=1$ and note that $\lambda_{p}^{(1)}=\overline{2 t}$ and $\left|\lambda_{p+1}^{(1)}\right| \geq 2 t+3$, then $\lambda_{p+1}^{(1)}=\overline{2 t+3}($ or $2 t+4)$, this part will become $2 t+3$ (or $\overline{2 t+4}$ ) in $\mu$, and by the definition of Göllnitz-Gordon 
marking, we see that $2 t+3$ (or $\overline{2 t+4}$ ) in $\mu$ is also marked with 1 .

(2) If $r \geq 2$, then we shall first show that there is no $r$-marked $\overline{2 t+3}$ in $\mu$. By the definition of $\lambda$, we see that the marks of the parts of size $2 t+2$ in $\lambda$ are greater than $r$. It follows that $\overline{2 t+3}$ in $\lambda$ is marked with 1 , and so there is no $r$-marked $\overline{2 t+3}$ in $\mu$. If there is an $r$-marked $2 t+4$ in $\lambda$, then there are 2 -marked, $\ldots,(r-1)$-marked parts $2 t+4$ and a 1 -marked $\overline{2 t+3}$ or $2 t+4$ in $\lambda$. Note that $\lambda_{p+1}^{(1)}=\overline{2 t+3}$ or $2 t+4$ will become $2 t+3$ or $\overline{2 t+4}$ in $\mu$, which is also marked with 1 in $\mu$. Moreover, there is no 1 -marked $\overline{2 t+2}$ or $2 t+2$ or $\overline{2 t+3}$ in $\mu$, and $r$ is the smallest mark of the parts $2 t+2$ in $\mu$, so the $r$-marked part $2 t+4$ in $\lambda$ will also be marked with $r$ in $\mu$.

Hence we prove that the assertion holds for parts of size greater than $2 t+2$ in $\mu$. So we conclude that $\mu$ is an overpartition in $\mathbb{F}_{N_{1}, \ldots, N_{k-1} ; i}$.

Let $\mu^{(1)}=\left(\mu_{1}^{(1)}, \mu_{2}^{(1)}, \ldots, \mu_{N_{1}}^{(1)}\right)$ be the first sub-overpartition of $\mu$. From the above proof, we see that $\lambda_{j}^{(1)}=\mu_{j}^{(1)}$ for $j \neq p, p+1$. Furthermore, $\mu_{p}^{(1)}$ is a non-overlined even part, $\mu_{p+1}^{(1)}$ is a non-overlined odd part or an overlined even part, and $\mu_{j}^{(1)}$ is an overlined odd part or a non-overlined even part, where $p+2 \leq j \leq N_{1}$. Again, by the above proof, we see that there is no overlined odd part of size $\left|\mu_{p}^{(1)}\right|+1$ in $\mu$. This proves that $\mu \in \overline{\mathbb{F}}_{N_{1}, \ldots, N_{k-1} ; i, p}^{(3)}$.

To prove that $\Phi_{3, p}$ is a bijection, we construct the inverse map $\Psi_{3, p}$ of $\Phi_{3, p}$. Let $\mu^{(1)}=$ $\left(\mu_{1}^{(1)}, \mu_{2}^{(1)}, \ldots, \mu_{N_{1}}^{(1)}\right)$ be the first sub-overpartition of $\mu$ in $\overline{\mathbb{F}}_{N_{1}, \ldots, N_{k-1} ; i, p}^{(3)}$. By definition, we see that $\mu_{p}^{(1)}$ is a non-overlined even part and $\mu_{p+1}^{(1)}$ is a non-overlined odd part or an overlined even part. Let $\mu_{p}^{(1)}=2 t$ and $\mu_{p+1}^{(1)}=2 a+1$ or $\overline{2 a+2}$. Note that $p>1$, so $t \geq 2$. By the definition of Göllnitz-Gordon marking, we see that $a \geq t$. Since $\mu \in \overline{\mathbb{F}}_{N_{1}, \ldots, N_{k-1} ; i, p}^{(3)}$, $\overline{2 t+1}$ does not occur in $\mu$.

To define $\Psi_{3, p}$, we define an index $r^{\prime}$ related to the sizes of $\mu_{p}^{(1)}=2 t$ and $\mu_{p+1}^{(1)}=2 a+1$ or $\overline{2 a+2}$. If $a=t$, or $a>t$ and $2 t+2$ does not occur in $\mu$, then set $r^{\prime}=1$; If $a>t$ and $2 t+2$ occurs in $\mu$, then set $r^{\prime}$ to be the smallest mark of the parts $2 t+2$ in $\mu$.

For $1<p \leq N_{1}$, define $\lambda=\Psi_{3, p}(\mu)$ which is obtained from $\mu$ by doing the following two operations.

(1) When $p=N_{1}$, we shall do nothing; When $1<p<N_{1}$, replace $\mu_{p+1}^{(1)}$ by $\overline{2 a+1}$ (or $2 a+2)$ if $\mu_{p+1}^{(1)}=2 a+1$ (or $\overline{2 a+2}$ ).

(2) When $r^{\prime}=1$, replace $\mu_{p}^{(1)}=2 t$ by $\overline{2 t-2}$; When $r^{\prime} \geq 2$, replace the $r^{\prime}$-marked $2 t+2$ in $\mu$ by $2 t$ and replace $\mu_{p}^{(1)}=2 t$ by $\overline{2 t}$.

Obviously, $|\mu|=|\lambda|+2$. It can be proved that $\lambda=\Psi_{3, p}(\mu) \in \mathbb{F}_{N_{1}, \ldots, N_{k-1} ; i, p}^{(3)}$ and $\Psi_{3, p}$ is the inverse map of $\Phi_{3, p}$. So, we conclude that $\Phi_{3, p}$ is a bijection between $\mathbb{F}_{N_{1}, \ldots, N_{k-1} ; i, p}^{(3)}$ and $\overline{\mathbb{F}}_{N_{1}, \ldots, N_{k-1} ; i, p}^{(3)}$. This completes the proof. 
For example, for $p=5$, let

$$
G G(\lambda)=\left[\begin{array}{lllllllll} 
& & & 4 & & \mathbf{1 0} & & 14 \\
& 2 & & 4 & & \overline{7} & 10 & & 14 \\
\overline{1} & & 3^{2} & & 6 & & \overline{\mathbf{1 0}} & \overline{\mathbf{1 3}} &
\end{array}\right] \begin{aligned}
& 3 \\
& 1
\end{aligned}
$$

be the Göllnitz-Gordon marking representation of $\lambda$ in $\mathbb{F}_{6,5,3 ; 3,5}^{(3)}$. It can be checked that $r=3$. Applying the bijection $\Phi_{3,5}$ to $\lambda$, we get

$$
G G(\mu)=\left[\begin{array}{lllllllll} 
& & & 4 & & & & \mathbf{1 2} & 14 \\
& 2 & & 4 & & \overline{7} & 10 & & 14 \\
\overline{1} & & 3^{2} & & 6 & & \mathbf{1 0} & \mathbf{1 3} &
\end{array}\right] \begin{aligned}
& 3 \\
& 2 \\
& 1
\end{aligned},
$$

which is in $\overline{\mathbb{F}}_{6,5,3 ; 3,5}^{(3)}$. Applying $\Psi_{3,5}$ to $\mu$, we see that $r^{\prime}=3$ and $\Psi_{3,5}(\mu)=\lambda$.

For another example, for $p=5$, let

$$
G G(\lambda)=\left[\begin{array}{rrrrrrr} 
& 4 & & & 14 \\
& 2 & 4 & & 8 & & 14 \\
\overline{1} & & \overline{4} & 7^{2} & \overline{8} & \overline{\mathbf{1 3}}
\end{array}\right] \begin{aligned}
& 3 \\
& 1
\end{aligned}
$$

be the Göllnitz-Gordon marking representation of $\lambda$ in $\mathbb{F}_{6,4,2 ; 3,5}^{(3)}$. Applying the bijection $\Phi_{3,5}$ to $\lambda$, we see that $r=1$ and

$$
G G(\mu)=\left[\begin{array}{lrrrrrr} 
& 4 & & & & 14 \\
& 2 & 4 & & 8 & & \\
\overline{1} & & \overline{4} & 7^{2} & \mathbf{1 0} & \mathbf{1 3}
\end{array}\right] \begin{aligned}
& 3 \\
& 2 \\
& 1
\end{aligned},
$$

which is in $\overline{\mathbb{F}}_{6,4,2 ; 3,5}^{(3)}$. Applying $\Psi_{3,5}$ to $\mu$, we obtain that $r^{\prime}=1$ and $\Psi_{3,5}(\mu)=\lambda$.

Lemma 4.7. For $1<p \leq N_{1}$, there is a bijection $\Phi_{4, p}$ between $\mathbb{F}_{N_{1}, \ldots, N_{k-1} ; i, p}^{(4)}$ and $\overline{\mathbb{F}}_{N_{1}, \ldots, N_{k-1} ; i, p}^{(4)}$. Furthermore, for $\lambda \in \mathbb{F}_{N_{1}, \ldots, N_{k-1} ; i, p}^{(4)}$ and $\mu=\Phi_{4, p}(\lambda) \in \overline{\mathbb{F}}_{N_{1}, \ldots, N_{k-1} ; i, p}^{(4)}$, we have

$$
|\mu|=|\lambda|+2, \quad \text { and } \quad \lambda_{j}^{(1)}=\mu_{j}^{(1)} \quad \text { for } j \neq p, p+1 \text {. }
$$

Proof. Let $\lambda^{(1)}=\left(\lambda_{1}^{(1)}, \lambda_{2}^{(1)}, \ldots, \lambda_{N_{1}}^{(1)}\right)$ be the first sub-overpartition of $\lambda$ in $\mathbb{F}_{N_{1}, \ldots, N_{k-1} ; i, p}^{(4)}$. By definition, we see that $\lambda_{p}^{(1)}$ is an overlined even part, set $\lambda_{p}^{(1)}=\overline{2 t}$. So $\overline{2 t+1}$ occurs in $\lambda$, assume that it is marked with $s$ in $\lambda$. From the definition of Göllnitz-Gordon marking, it follows that $s \geq 2$. Note that $\lambda_{j}^{(1)}$ is an overlined odd part or a non-overlined even part, where $p+1 \leq j \leq N_{1}$, set $\lambda_{p+1}^{(1)}=\overline{2 a+1}$ or $2 a+2$, by the definition of Göllnitz-Gordon marking, we see that $a \geq t+1$.

To define $\Phi_{4, p}$, we also need to use the index $r$ defined in the bijection $\Phi_{3, p}$. Recall that if $\lambda_{p-1}^{(1)}=\overline{2 t-2}$, or $2 t-2$, or $\overline{2 t-1}$, or $2 t-1$, then $r=1$. If $\left|\lambda_{p-1}\right| \leq 2 t-3$ and 
there exists an integer $b$ such that there are $b$-marked parts $2 t-2$ and $2 t$ in $\lambda$, then $r=b$. Otherwise, set $r$ to be the largest mark of the parts of size $2 t$ in $\lambda$. By definition, we see that $s>r \geq 1$.

For $1<p \leq N_{1}$, define $\mu=\Phi_{4, p}(\lambda)$ which can be obtained from $\lambda$ by doing the following two operations.

(1) When $r=1$, replace $\lambda_{p}^{(1)}=\overline{2 t}$ by $\overline{2 t+1}$ and replace the $s$-marked $\overline{2 t+1}$ in $\lambda$ by $2 t+2$; When $r \geq 2$, first replace $\lambda_{p}^{(1)}=\overline{2 t}$ by $2 t$, and then replace the $r$-marked $2 t$ in $\lambda$ by $\overline{2 t+1}$ and the $s$-marked $\overline{2 t+1}$ in $\lambda$ by $2 t+2$.

(2) When $1<p<N_{1}$, replace $\lambda_{p+1}^{(1)}$ by $2 a+1$ (or $\overline{2 a+2}$ ) if $\lambda_{p+1}^{(1)}=\overline{2 a+1}($ or $2 a+2)$; When $p=N_{1}$, we shall do nothing.

Obviously, $|\mu|=|\lambda|+2$. We first show that $\mu$ is an overpartition in $\mathbb{F}_{N_{1}, \ldots, N_{k-1} ; i}$. We assert that the parts from $\lambda$ in $\mu$ have the same marks as in $\lambda$ and the new generated parts in $\mu$ replacing the parts in $\lambda$ have the same marks as their original parts in $\lambda$. By the definition of $\mu$, it is obvious that the marks of parts of size not exceed $2 t-1$ in $\mu$ stay the same as in $\lambda$. We proceed to show that the marks of parts of size $2 t$ and $2 t+1$ from $\lambda$ in $\mu$ are the same as in $\lambda$, the new generated part $\overline{2 t+1}$ replacing $\lambda_{p}^{(1)}=\overline{2 t}$ is marked with 1 when $r=1$, and the new generated part $2 t$ replacing $\lambda_{p}^{(1)}=\overline{2 t}$ is marked with 1 and the new generated part $\overline{2 t+1}$ replacing the $r$-marked $2 t$ in $\lambda$ is marked with $r$ when $r \geq 2$. It should be mentioned that $2 t+1$ does not occur in $\lambda$. We consider the following two cases:

(1) When $r=1$, in this case, we see that $2 t-2 \leq\left|\lambda_{p-1}^{(1)}\right| \leq 2 t-1$, or $\left|\lambda_{p-1}\right| \leq 2 t-3$ and there is only one part of size $2 t$ in $\lambda$. There are two subcases:

(1.1) When $\lambda_{p-1}^{(1)}=\overline{2 t-2}$, or $2 t-2$, or $\overline{2 t-1}$, or $2 t-1$, by the definition of GöllnitzGordon marking, we see that the marks of parts $2 t$ from $\lambda$ in $\mu$ are the same as in $\lambda$. Note that $\left|\lambda_{p-1}^{(1)}\right| \leq 2 t-1$, so the new generated part $\overline{2 t+1}$ in $\mu$ replacing $\lambda_{p}^{(1)}$ should be marked with 1 .

(1.2) When $\left|\lambda_{p-1}\right| \leq 2 t-3$ and there is only one part of size $2 t$ in $\lambda$, that is, $\lambda_{p}^{(1)}=\overline{2 t}$, there are no parts of size $2 t$ in $\mu$, so the new generated part $\overline{2 t+1}$ in $\mu$ replacing $\lambda_{p}^{(1)}=\overline{2 t}$ is marked with 1 .

(2) When $r \geq 2$, either $\left|\lambda_{p-1}^{(1)}\right| \leq 2 t-3$ and there are $r$-marked parts $2 t-2$ and $2 t$ in $\lambda$, or $r$ is the largest mark of the parts $2 t$ in $\lambda$. By the definition of Göllnitz-Gordon marking, we see that there are $f$-marked parts of size $2 t$ in $\lambda$, where $1 \leq f \leq r$. Note that $\left|\lambda_{p-1}\right| \leq 2 t-3$, it follows that the new generated part $2 t$ replacing $\lambda_{p}^{(1)}=\overline{2 t}$ is marked with 1 and the marks of parts $2 t$ from $\lambda$ in $\mu$ stay the same as in $\lambda$. Hence there are $f$-marked 2t's in $\mu$, where $1 \leq f \leq r-1$. Therefore the new generated part $\overline{2 t+1}$ replacing the $r$-marked $2 t$ in $\lambda$ is marked with $r$ in $\mu$.

Next, we show that the new generated part $2 t+2$ in $\mu$ replacing the $s$-marked $\overline{2 t+1}$ in $\lambda$ is marked with $s$ and the marks of parts $2 t+2$ from $\lambda$ in $\mu$ are the same as in $\lambda$. Since there is an $s$-marked $\overline{2 t+1}$ in $\lambda$, there are $f$-marked parts of size $2 t$ in $\lambda$, where 
$1 \leq f \leq s-1$. From the preceding proof and the definition of $\mu$, it follows that there are $f$-marked 2t's in $\mu$ where $1 \leq f \leq s-1$ and $f \neq r$, and there is an $r$-marked $\overline{2 t+1}$ in $\mu$. Hence the new generated part $2 t+2$ in $\mu$ replacing the $s$-marked $\overline{2 t+1}$ in $\lambda$ should be marked with $s$. Furthermore, the marks of parts $2 t+2$ from $\lambda$ in $\mu$ are the same as in $\lambda$.

It remains to show that the marks of parts of size greater than $2 t+2$ in $\mu$ stay the same as in $\lambda$. By the definition of Göllnitz-Gordon marking, it is easy to see that the new generated $s$-marked $2 t+2$ in $\mu$ replacing the $s$-marked $\overline{2 t+1}$ in $\lambda$ only affect the mark of the $s$-marked part of size $2 t+3$ or $2 t+4$ in $\mu$. Since $s \geq 2$, we see that there is no $s$-marked $\overline{2 t+3}$ in $\lambda$, and so there is no $s$-marked $\overline{2 t+3}$ in $\mu$. Hence it suffices to show that the $s$-marked $2 t+4$ in $\lambda$ is also marked with $s$ in $\mu$ even if the $s$-marked $\overline{2 t+1}$ in $\lambda$ is replaced by the $s$-marked $2 t+2$ in $\mu$.

Note that there is an s-marked $\overline{2 t+1}$ in $\lambda$, so there are a 1-marked $\overline{2 t}$ and $f$-marked $2 t$ 's in $\lambda$, where $2 \leq f \leq s-1$. It follows that the marks of the parts $2 t+2$ in $\lambda$ are greater than $s$. Hence we conclude that if there exists an $s$-marked $2 t+4$ in $\lambda$, then there are a 1 -marked $\overline{2 t+3}$ or $2 t+4$ and $f$-marked (2t+4)'s in $\lambda$, where $2 \leq f \leq s-1$. Note that $\lambda_{p+1}^{(1)}=\overline{2 t+3}$ or $2 t+4$ will become $2 t+3$ or $\overline{2 t+4}$ in $\mu$, which is also marked with 1 in $\mu$. Moreover, there is no 1-marked $\overline{2 t+2}$ or $2 t+2$ or $\overline{2 t+3}$ in $\mu$, and $s$ is the least mark of the parts $2 t+2$ in $\mu$, so by the definition of Göllnitz-Gordon marking, we see that the $s$-marked $2 t+4$ in $\lambda$ will also be marked with $s$ in $\mu$.

Thus, we have shown that the marks of parts in $\mu$ are the same as the marks of their original parts in $\lambda$. Hence $\mu$ is an overpartition in $\mathbb{F}_{N_{1}, \ldots, N_{k-1} ; i}$.

Let $\mu^{(1)}=\left(\mu_{1}^{(1)}, \mu_{2}^{(1)}, \ldots, \mu_{N_{1}}^{(1)}\right)$ be the first sub-overpartition of $\mu$. It can be seen from the above proof that $\lambda_{j}^{(1)}=\mu_{j}^{(1)}$ for $j \neq p, p+1$ and $\mu_{p}^{(1)}$ is an overlined odd part or a non-overlined even part. Furthermore, if $\mu_{p}^{(1)}$ is an overlined odd part, then there is a nonoverlined even part of size $\left|\mu_{p}^{(1)}\right|+1$ in $\mu$, and $\left|\mu_{p+1}^{(1)}\right| \geq\left|\mu_{p}^{(1)}\right|+2$. If $\mu_{p}^{(1)}$ is a non-overlined even part, then there are an overlined odd part of size $\left|\mu_{p}^{(1)}\right|+1$ and a non-overlined even part of size $\left|\mu_{p}^{(1)}\right|+2$ in $\mu$, and $\left|\mu_{p+1}^{(1)}\right|>\left|\mu_{p}^{(1)}\right|+2$. Moreover, it is easy to see that $\mu_{p+1}^{(1)}$ is a non-overlined odd part or an overlined even part, and $\mu_{j}^{(1)}$ is an overlined odd part or a non-overlined even part, where $p+2 \leq j \leq N_{1}$. This proves that $\mu$ is an overpartition in $\overline{\mathbb{F}}_{N_{1}, \ldots, N_{k-1} ; i, p}^{(4)}$.

We proceed to construct the inverse map $\Psi_{4, p}$ of $\Phi_{4, p}$, where $1<p \leq N_{1}$. Let $\mu^{(1)}=$ $\left(\mu_{1}^{(1)}, \mu_{2}^{(1)}, \ldots, \mu_{N_{1}}^{(1)}\right)$ be the first sub-overpartition of $\mu$ in $\overline{\mathbb{F}}_{N_{1}, \ldots, N_{k-1} ; i, p}^{(4)}$ By definition, we see that $\mu_{p}^{(1)}$ is an overlined odd part or a non-overlined even part and $\mu_{p+1}^{(1)}$ is a non-overlined odd part or an overlined even part. If $\mu_{p}^{(1)}$ is an overlined odd part, set $\mu_{p}^{(1)}=\overline{2 t+1}$ and $\mu_{p+1}^{(1)}=2 a+1$ or $\overline{2 a+2}$, then by definition, we see that $2 t+2$ occurs in $\mu$ and $a \geq t+1$. If $\mu_{p}^{(1)}$ is a non-overlined even part, set $\mu_{p}^{(1)}=2 t$ and $\mu_{p+1}^{(1)}=2 a+1$ or $\overline{2 a+2}$, then $\overline{2 t+1}$ and $2 t+2$ occur in $\mu$ and $a \geq t+1$.

Let $r^{\prime}$ be the mark of $\overline{2 t+1}$ in $\mu$ and $s^{\prime}$ be the smallest mark of the parts $2 t+2$ in 
$\mu$. It follows from the definition of Göllnitz-Gordon marking that $s^{\prime}>r^{\prime} \geq 1$. Define $\lambda=\Psi_{4, p}(\mu)$ which is obtained from $\mu$ by doing the following two operations.

(1) When $p=N_{1}$, we shall do nothing; When $p<N_{1}$, replace $\mu_{p+1}^{(1)}$ by $\overline{2 a+1}$ (or $2 a+2)$ if $\mu_{p+1}^{(1)}=2 a+1$ (or $\overline{2 a+2}$ ).

(2) When $r^{\prime}=1$, replace $\mu_{p}^{(1)}=\overline{2 t+1}$ by $\overline{2 t}$ and replace the $s^{\prime}$-marked $2 t+2$ in $\mu$ by $\overline{2 t+1}$. When $r^{\prime} \geq 2$, replace $\mu_{p}^{(1)}=2 t$ by $\overline{2 t}$, replace the $r^{\prime}$-marked $\overline{2 t+1}$ in $\mu$ by $2 t$ and replace the $s^{\prime}$-marked $2 t+2$ in $\mu$ by $\overline{2 t+1}$.

It can be verified that $\lambda=\Psi_{4, p}(\mu) \in \mathbb{F}_{N_{1}, \ldots, N_{k-1} ; i, p}^{(4)}$ and $|\mu|=|\lambda|+2$, and $\Psi_{4, p}$ is the inverse map of $\Phi_{4, p}$. So, we conclude that $\Phi_{4, p}$ is a bijection between $\mathbb{F}_{N_{1}, \ldots, N_{k-1} ; i, p}^{(4)}$ and $\overline{\mathbb{F}}_{N_{1}, \ldots, N_{k-1} ; i, p}^{(4)}$. This completes the proof.

For example, for $p=4$, let

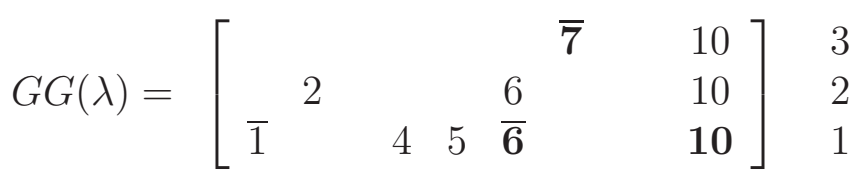

be the Göllnitz-Gordon marking representation of $\lambda$ in $\mathbb{F}_{5,3,2 ; 3,4}^{(4)}$. Applying the bijection $\Phi_{4,4}$ to $\lambda$, we see that $r=1, s=3$, and

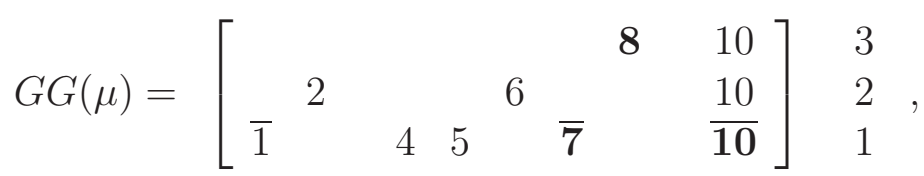

which is in $\overline{\mathbb{F}}_{5,3,2 ; 3,4}^{(4)}$. Applying $\Psi_{4,4}$ to $\mu$, we have $r^{\prime}=1, s^{\prime}=3$, and $\Psi_{4,4}(\mu)=\lambda$.

For example, for $p=3$, let

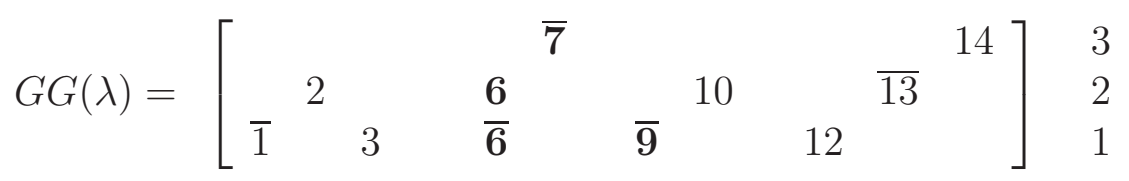

be the Göllnitz-Gordon marking representation of $\lambda$ in $\mathbb{F}_{5,4,2 ; 3,3}^{(4)}$. Applying the bijection $\Phi_{4,3}$ to $\lambda$, we see that $r=2, s=3$, and

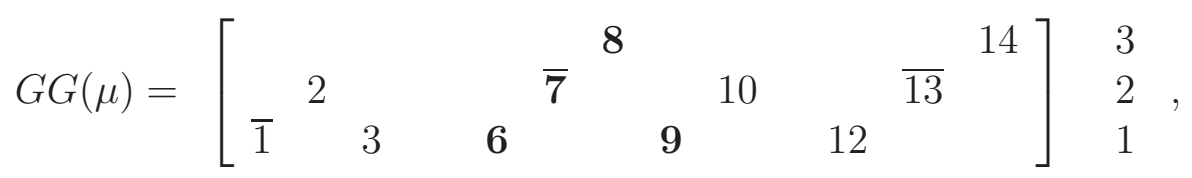

which is in $\overline{\mathbb{F}}_{5,4,2 ; 3,3}^{(4)}$. Applying $\Psi_{4,3}$ to $\mu$, we have $r^{\prime}=2, s^{\prime}=3$, and $\Psi_{4,3}(\mu)=\lambda$.

We conclude this section by giving a proof of Lemma 4.2 . 
Proof of Lemma 4.2. Supposed that $k \geq i \geq 1, N_{1} \geq N_{2} \geq \cdots \geq N_{k-1} \geq 0$ and $1<p \leq N_{1}$. From the definitions of $\mathbb{F}_{N_{1}, \ldots, N_{k-1} ; i, p}^{(l)}$ and $\overline{\mathbb{F}}_{N_{1}, \ldots, N_{k-1} ; i, p}^{(l)}$, where $1 \leq l \leq 4$, we have

$$
\mathbb{F}_{N_{1}, \ldots, N_{k-1} ; i, p}=\bigcup_{l=1}^{4} \mathbb{F}_{N_{1}, \ldots, N_{k-1} ; i, p}^{(l)}
$$

and

$$
\overline{\mathbb{F}}_{N_{1}, \ldots, N_{k-1} ; i, p}=\bigcup_{l=1}^{4} \overline{\mathbb{F}}_{N_{1}, \ldots, N_{k-1} ; i, p}^{(l)} .
$$

Let $\lambda \in \mathbb{F}_{N_{1}, \ldots, N_{k-1} ; i, p}$, define

$$
\mu=\Phi_{p}(\lambda)= \begin{cases}\Phi_{1, p}(\lambda), & \text { if } \lambda \in \mathbb{F}_{N_{1}, \ldots, N_{k-1} ; i, p}^{(1)} \\ \Phi_{2, p}(\lambda), & \text { if } \lambda \in \mathbb{F}_{N_{1}, \ldots, N_{k-1} ; i, p}^{(2)} \\ \Phi_{3, p}(\lambda), & \text { if } \lambda \in \mathbb{F}_{N_{1}, \ldots, N_{k-1} ; i, p}^{(3)} \\ \Phi_{4, p}(\lambda), & \text { if } \lambda \in \mathbb{F}_{N_{1}, \ldots, N_{k-1} ; i, p}^{(4)}\end{cases}
$$

Combining Lemma 4.4, Lemma 4.5, Lemma 4.6 and Lemma 4.7, we conclude that $\Phi_{p}$ is a bijection between $\mathbb{F}_{N_{1}, \ldots, N_{k-1} ; i, p}$ and $\overline{\mathbb{F}}_{N_{1}, \ldots, N_{k-1} ; i, p}$. Furthermore, for $\lambda \in \mathbb{F}_{N_{1}, \ldots, N_{k-1} ; i, p}$ and $\mu=\Phi_{p}(\lambda) \in \overline{\mathbb{F}}_{N_{1}, \ldots, N_{k-1} ; i, p}$, we have

$$
|\mu|=|\lambda|+2, \quad \text { and } \quad \lambda_{j}^{(1)}=\mu_{j}^{(1)} \quad \text { for } \quad j \neq p, p+1 .
$$

\section{Proof of Lemma 3.6}

Let $\mathbb{D}_{N}$ denote the set of partitions $\eta=\left(\eta_{1}, \eta_{2}, \ldots, \eta_{\ell}\right)$ with distinct negative odd parts which lay in $[1-2 N,-1]$, that is, $\eta_{j}$ is negative and odd for $1 \leq j \leq \ell$ and $1-2 N \leq \eta_{1}<$ $\eta_{2}<\cdots<\eta_{\ell} \leq-1$. It is clear to see that the generating function of partitions in $\mathbb{D}_{N}$ :

$$
\sum_{\eta \in \mathbb{D}_{N}} q^{|\eta|}=\left(1+q^{1-2 N}\right)\left(1+q^{3-2 N}\right) \cdots\left(1+q^{-1}\right)=\left(-q^{1-2 N} ; q^{2}\right)_{N} .
$$

Hence Lemma 3.6 is equivalent to the following combinatorial statement.

Theorem 5.1. For $k \geq i \geq 1$ and $N_{1} \geq N_{2} \geq \cdots \geq N_{k-1} \geq 0$, there is a bijection $\Theta$ between $\mathbb{G}_{N_{1}, \ldots, N_{k-1} ; i}$ and $\mathbb{D}_{N_{1}} \times \mathbb{E}_{N_{1}, \ldots, N_{k-1} ; i}$ such that for $\mu \in \mathbb{G}_{N_{1}, \ldots, N_{k-1} ; i}$ and $\Theta(\mu)=$ $(\eta, \nu) \in \mathbb{D}_{N_{1}} \times \mathbb{E}_{N_{1}, \ldots, N_{k-1} ; i}$, we have $|\mu|=|\eta|+|\nu|$. 
Let $\mu^{(r)}=\left(\mu_{1}^{(r)}, \mu_{2}^{(r)}, \ldots, \mu_{N_{r}}^{(r)}\right)$ be the $r$-th sub-overpartition of $\mu$ in $\mathbb{G}_{N_{1}, \ldots, N_{k-1} ; i}$, where $1 \leq r \leq k-1$. From the definition of $\mathbb{G}_{N_{1}, \ldots, N_{k-1} ;}$, it is easy to see that $\mu_{j}^{(r)}$ is an overlined odd part or a non-overlined even part for $1 \leq r \leq k-1$ and $1 \leq j \leq N_{r}$. Observe that $\mathbb{E}_{N_{1}, \ldots, N_{k-1} ; i}$ is the set of overpartitions in $\mathbb{G}_{N_{1}, \ldots, N_{k-1} ; i}$ for which there are no overlined odd parts, so the key point in the construction of the bijection $\Theta$ is to remove all overlined odd parts of an overpartition in $\mathbb{G}_{N_{1}, \ldots, N_{k-1} ; i}$ to get a new overpartition in $\mathbb{E}_{N_{1}, \ldots, N_{k-1} ; i}$.

Similarly to the bijection $\Phi$ in Section 4 , we will define three subsets $\mathbb{G}_{N_{1}, \ldots, N_{k-1} ; i, p}$, $\overline{\mathbb{G}}_{N_{1}, \ldots, N_{k-1} ; i, p}$ and $\overrightarrow{\mathbb{G}}_{N_{1}, \ldots, N_{k-1} ; i, p}$ of $\mathbb{G}_{N_{1}, \ldots, N_{k-1} ; i}$. Then we build a bijection $\Theta_{p}$ between $\mathbb{G}_{N_{1}, \ldots, N_{k-1} ; i, p}$ and $\overline{\mathbb{G}}_{N_{1}, \ldots, N_{k-1} ; i, p}$ and a bijection $\Theta_{(p)}$ between $\mathbb{G}_{N_{1}, \ldots, N_{k-1} ; i, p}$ and $\overrightarrow{\mathbb{G}}_{N_{1}, \ldots, N_{k-1} ; i, p}$. Similarly, $\Theta_{(p)}$ can be obtained by successively using the bijection $\Theta_{p}$ and plays a crucial role in the construction of the bijection $\Theta$ in Theorem 5.1 .

Let $\mu^{(1)}=\left(\mu_{1}^{(1)}, \mu_{2}^{(1)}, \ldots, \mu_{N_{1}}^{(1)}\right)$ be the first sub-overpartition of $\mu$ in $\mathbb{G}_{N_{1}, \ldots, N_{k-1} ; i}$. To define the above three subsets of $\mathbb{G}_{N_{1}, \ldots, N_{k-1} ; i}$, we divide the parts in $\mu^{(1)}$ into two classes. A part $\mu_{j}^{(1)}$ is of type $\mathrm{O}$ if $\mu_{j}^{(1)}$ is an overlined odd part or there is an overlined odd part of size $\left|\mu_{j}^{(1)}\right|+1$ in $\mu$, and a part $\mu_{j}^{(1)}$ is of type $\mathrm{E}$ if $\mu_{j}^{(1)}$ is a non-overlined even part and there is no overlined odd part of size $\left|\mu_{j}^{(1)}\right|+1$ in $\mu$. We say that two parts $\mu_{j_{1}}^{(1)}$ and $\mu_{j_{2}}^{(1)}$ are of the same type if they are both of type $\mathrm{O}$ or they are both of type E. For example, let

$$
G G(\mu)=\left[\begin{array}{l}
\mu^{(3)} \\
\mu^{(2)} \\
\mu^{(1)}
\end{array}\right]=\left[\begin{array}{llllllllll} 
& & 4 & & & & & & 10 & \\
& 2 & & 6 & & \overline{9} & & 12 & & \\
\overline{1} & & 4 & & 8 & & 12 & & 16
\end{array}\right] \begin{aligned}
& 3 \\
& 2 \\
& 1
\end{aligned}
$$

be the Göllnitz-Gordon marking representation of $\mu$ in $\mathbb{G}_{5,4,3 ; 3}$. From definition, we see that the parts $\mu_{1}^{(1)}=\overline{1}$ and $\mu_{3}^{(1)}=8$ are of type $\mathrm{O}$, and the parts $\mu_{2}^{(1)}=4, \mu_{4}^{(1)}=12$ and $\mu_{5}^{(1)}=16$ are of type E.

Let $k \geq i \geq 1$ and $N_{1} \geq N_{2} \geq \cdots \geq N_{k-1} \geq 0$ be given. For $1 \leq p \leq N_{1}$, the subsets $\mathbb{G}_{N_{1}, \ldots, N_{k-1} ; i, p}, \overline{\mathbb{G}}_{N_{1}, \ldots, N_{k-1} ; i, p}$ and $\overrightarrow{\mathbb{G}}_{N_{1}, \ldots, N_{k-1} ; i, p}$ are described by using the first sub-overpartition $\mu^{(1)}=\left(\mu_{1}^{(1)}, \mu_{2}^{(1)}, \ldots, \mu_{N_{1}}^{(1)}\right)$ of an overpartition $\mu$ in $\mathbb{G}_{N_{1}, \ldots, N_{k-1} ; i}$, where $\mu_{1}^{(1)} \leq \mu_{2}^{(1)} \leq \cdots \leq \mu_{N_{1}}^{(1)}$.

$\diamond$ Let $\mathbb{G}_{N_{1}, \ldots, N_{k-1} ; i, p}$ be the set of overpartitions $\mu$ in $\mathbb{G}_{N_{1}, \ldots, N_{k-1} ; i}$ such that $(1) \mu_{p}^{(1)}$ is of type $\mathrm{O}$ for $1 \leq p \leq N_{1}$; (2) $\mu_{j}^{(1)}$ is of type E, where $p+1 \leq j \leq N_{1}$.

$\diamond$ Let $\overline{\mathbb{G}}_{N_{1}, \ldots, N_{k-1} ; i, p}$ be the set of overpartitions $\mu$ in $\mathbb{G}_{N_{1}, \ldots, N_{k-1} ; i}$ such that (1) $\mu_{p}^{(1)}$ is of type $\mathrm{E} ;(2) \mu_{p+1}^{(1)}$ is of type $\mathrm{O} ;(3) \mu_{j}^{(1)}$ is of type E, where $p+2 \leq j \leq N_{1}$.

$\diamond$ Let $\overrightarrow{\mathbb{G}}_{N_{1}, \ldots, N_{k-1} ; i, p}$ be the set of overpartitions $\mu$ in $\mathbb{G}_{N_{1}, \ldots, N_{k-1} ; i}$ such that $\mu_{j}^{(1)}$ is of type E, where $p \leq j \leq N_{1}$.

By definition, it is easy to see that for $1 \leq p \leq N_{1}-2$

$$
\overline{\mathbb{G}}_{N_{1}, \ldots, N_{k-1} ; i, p} \subseteq \mathbb{G}_{N_{1}, \ldots, N_{k-1} ; i, p+1} \subseteq \overrightarrow{\mathbb{G}}_{N_{1}, \ldots, N_{k-1} ; i, p+2} .
$$


Lemma 5.2. For $1 \leq p \leq N_{1}$, there is a bijection $\Theta_{p}$ between $\mathbb{G}_{N_{1}, \ldots, N_{k-1} ; i, p}$ and $\overline{\mathbb{G}}_{N_{1}, \ldots, N_{k-1} ; i, p}$. Furthermore, for $\mu \in \mathbb{G}_{N_{1}, \ldots, N_{k-1} ; i, p}$ and $\nu=\Theta_{p}(\mu) \in \overline{\mathbb{G}}_{N_{1}, \ldots, N_{k-1} ; i, p}$, we have $\mu_{j}^{(1)}$ and $\nu_{j}^{(1)}$ are of the same type for $j \neq p, p+1$, and $|\nu|=|\mu|+2-\delta_{p}^{N_{1}}$, where

$$
\delta_{p}^{N_{1}}= \begin{cases}1, & \text { if } p=N_{1} \\ 0, & \text { if } p \neq N_{1}\end{cases}
$$

Applying in succession the bijection in Lemma 5.2 leads to a bijection between $\mathbb{G}_{N_{1}, \ldots, N_{k-1} ; i, p}$ and $\overrightarrow{\mathbb{G}}_{N_{1}, \ldots, N_{k-1} ; i, p}$.

Lemma 5.3. For $1 \leq p \leq N_{1}$, there is a bijection $\Theta_{(p)}$ between $\mathbb{G}_{N_{1}, \ldots, N_{k-1} ;, p}$ and $\overrightarrow{\mathbb{G}}_{N_{1}, \ldots, N_{k-1} ; i, p}$. Furthermore, for $\mu \in \mathbb{G}_{N_{1}, \ldots, N_{k-1} ; i, p}$ and $\nu=\Theta_{(p)}(\mu) \in \overrightarrow{\mathbb{G}}_{N_{1}, \ldots, N_{k-1} ; i, p}$, we have $\mu_{j}^{(1)}$ and $\nu_{j}^{(1)}$ are of the same type for $j<p$ and $|\nu|=|\mu|+2 N_{1}-2 p+1$.

Proof. Define $\Theta_{(p)}=\Theta_{N_{1}} \Theta_{N_{1}-1} \cdots \Theta_{p}$, by Lemma 5.2, it is easy to verify that $\Theta_{(p)}$ is a bijection between $\mathbb{G}_{N_{1}, \ldots, N_{k-1} ; i, p}$ and $\overrightarrow{\mathbb{G}}_{N_{1}, \ldots, N_{k-1} ; i, p}$ as desired.

Before giving a proof of Lemma [5.2, we first give a proof of Lemma 3.6 by successively applying the bijection $\Theta_{(p)}$ in Lemma 5.3.

Proof of Lemma 3.6. It is equivalent to prove Theorem 5.1. Let $\mu$ be an overpartition in $\mathbb{G}_{N_{1}, \ldots, N_{k-1} ; i}$. We aim to define $\Theta(\mu)=(\eta, \nu)$ such that $|\eta|+|\nu|=|\mu|, \eta$ is a partition in $\mathbb{D}_{N_{1}}$ and $\nu$ is an overpartition in $\mathbb{E}_{N_{1}, \ldots, N_{k-1} ; i}$. We consider the following two cases.

Case 1. If there are no overlined odd parts in $\mu$, then set $\nu=\mu$ and $\eta=\emptyset$. It is easy to see that $\nu \in \mathbb{E}_{N_{1}, \ldots, N_{k-1} ; i}$ and $|\nu|=|\mu|$.

Case 2. If there are $s \geq 1$ overlined odd parts in $\mu$, then there are $s$ parts of type $\mathrm{O}$ in the first sub-overpartition of $\mu$. Note that if there is an overlined odd part in $\mu$, say $\overline{2 t+1}$, then it follows from the definition of Göllnitz-Gordon marking that there exists a 1 -marked $\overline{2 t+1}$ or $2 t$ in $\mu$. So, each overlined odd part in $\mu$ uniquely determines a part of type $\mathrm{O}$ in the first sub-overpartition of $\mu$.

Let $\mu_{j_{1}}^{(1)}, \mu_{j_{2}}^{(1)}, \ldots, \mu_{j_{s-1}}^{(1)}, \mu_{j_{s}}^{(1)}$ be the parts of type $\mathrm{O}$ in the first sub-overpartition $\mu^{(1)}=\left(\mu_{1}^{(1)}, \mu_{2}^{(1)}, \ldots, \mu_{N_{1}}^{(1)}\right)$ of $\mu$, where $1 \leq j_{1}<j_{2}<\cdots<j_{s} \leq N_{1}$. It is easy to see that $\mu \in \mathbb{G}_{N_{1}, \ldots, N_{k-1} ; i, j_{s}}$. Set

$$
\eta=\left(1-2\left(N_{1}-j_{1}+1\right), 1-2\left(N_{1}-j_{2}+1\right), \ldots, 1-2\left(N_{1}-j_{s}+1\right)\right) .
$$

Obviously, $\eta \in \mathbb{D}_{N_{1}}$. The partition $\nu$ can be obtained from $\mu$ by employing the bijection in Lemma $5.3 \mathrm{~s}$ times. We denote the intermediate partitions by $\gamma^{0}, \gamma^{1}, \ldots, \gamma^{s}$ with $\gamma^{0}=\mu$ and $\gamma^{s}=\nu$. For $1 \leq b \leq s$, the intermediate partition $\gamma^{b}$ can be obtained from $\gamma^{b-1}$ by using $\Theta_{\left(j_{s-b+1}\right)}$ in Lemma 5.3, that is, for $1 \leq b \leq s$,

$$
\gamma^{b}=\Theta_{\left(j_{s-b+1}\right)}\left(\gamma^{b-1}\right)
$$


Note that $\gamma^{0} \in \mathbb{G}_{N_{1}, \ldots, N_{k-1} ; i, j_{s}}$, so by Lemma 5.3, we see that

$$
\gamma^{1} \in \mathbb{G}_{N_{1}, \ldots, N_{k-1} ; i, j_{s-1}} \text { and }\left|\gamma^{1}\right|=|\mu|+2 N_{1}-2 j_{s}+1
$$

and the first $\left(j_{s}-1\right)$ parts in the first sub-overpartitions of $\gamma^{1}$ and $\gamma^{0}$ are of the same type.

Successively employing Lemma [5.3, we derive that for $1 \leq b \leq s-1$,

$$
\gamma^{b} \in \mathbb{G}_{N_{1}, \ldots, N_{k-1} ; i, j_{s-p}} \text { and }\left|\gamma^{b}\right|=|\mu|+\sum_{r=1}^{b}\left(2 N_{1}-2 j_{s-r+1}+1\right),
$$

and

$$
\gamma^{s} \in \overrightarrow{\mathbb{G}}_{N_{1}, \ldots, N_{k-1} ; i, j_{1}} \text { and }\left|\gamma^{s}\right|=|\mu|+\sum_{r=1}^{s}\left(2 N_{1}-2 j_{s-r+1}+1\right) .
$$

Furthermore, for $1 \leq b \leq s$, the first $\left(j_{s-b+1}-1\right)$ parts in the first sub-overpartitions of $\gamma^{b}$ and $\gamma^{0}$ are of the same type. From the definition of $\mu$, the first $\left(j_{1}-1\right)$ parts in the first sub-overpartition of $\mu$ are of type E, and by the definition of $\overrightarrow{\mathbb{G}}_{N_{1}, \ldots, N_{k-1} ; i, j_{1}}$, we derive that there are no overlined odd parts in $\gamma^{s}$. Hence

$$
\nu=\gamma^{s} \in \mathbb{E}_{N_{1}, \ldots, N_{k-1} ; i} \text { and }|\nu|=|\mu|+\sum_{r=1}^{s}\left(2 N_{1}-2 j_{s-r+1}+1\right) .
$$

It is easy to check that $|\eta|+|\nu|=|\mu|$. Therefore $\Theta$ is well-defined.

To prove that $\Theta$ is a bijection, we shall give a brief description of the inverse map $\Lambda$ of $\Theta$. Let $\nu$ be an overpartition in $\mathbb{E}_{N_{1}, \ldots, N_{k-1} ; i}$ and $\eta$ be a partition into distinct negative odd parts lying in $\left[1-2 N_{1},-1\right]$. We shall define $\Lambda(\eta, \nu)=\mu$ such that $\mu$ is an overpartition in $\mathbb{G}_{N_{1}, \ldots, N_{k-1} ; i}$ and $|\eta|+|\nu|=|\mu|$. There are two cases.

Case 1. If $\eta=\emptyset$, then set $\mu=\nu$. Note that $\mathbb{E}_{N_{1}, \ldots, N_{k-1} ; i} \subseteq \mathbb{G}_{N_{1}, \ldots, N_{k-1} ; i}$, so $\mu \in$ $\mathbb{G}_{N_{1}, \ldots, N_{k-1} ; i}$ and there are no overlined odd parts in $\mu$.

Case 2. If $\eta \neq \emptyset$ and assume that

$$
\eta=\left(1-2\left(N_{1}-j_{1}+1\right), 1-2\left(N_{1}-j_{2}+1\right), \ldots, 1-2\left(N_{1}-j_{s}+1\right)\right),
$$

where $1 \leq j_{1}<j_{2}<\cdots<j_{s} \leq N_{1}$, then $\mu$ can be recovered from $\nu$ by using the bijection in Lemma $5.3 s$ times. We denote the intermediate partitions by $\delta^{0}, \delta^{1}, \ldots, \delta^{s}$ with $\delta^{s}=\nu$ and $\delta^{0}=\mu$. For $1 \leq b \leq s$, the intermediate partition $\delta^{b-1}$ can be obtained from $\delta^{b}$ by using the bijection $\Theta_{\left(j_{s-b+1}\right)}^{-1}$ in Lemma 5.3, that is $\delta^{b-1}=\Theta_{\left(j_{s-b+1}\right)}^{-1}\left(\delta^{b}\right)$. Set $\mu=\delta^{0}$, by Lemma 5.3. we derive that $\mu$ is an overpartition in $\mathbb{G}_{N_{1}, \ldots, N_{k-1} ; i}$ and $|\mu|=|\eta|+|\nu|$, and $\Lambda(\Theta(\mu))=\mu$ for any $\mu$ in $\mathbb{G}_{N_{1}, \ldots, N_{k-1} ; i}$. Hence we conclude that $\Theta$ is a bijection between $\mathbb{G}_{N_{1}, \ldots, N_{k-1} ; i}$ and $\mathbb{D}_{N_{1}} \times \mathbb{E}_{N_{1}, \ldots, N_{k-1} ; i}$. This completes the proof of Theorem 5.1, and hence Lemma 3.6 is verified. 
We proceed to give a proof of Lemma 5.2.

Proof of Lemma [5.2. Let $\mu^{(1)}=\left(\mu_{1}^{(1)}, \mu_{2}^{(1)}, \ldots, \mu_{N_{1}}^{(1)}\right)$ be the first sub-overpartition of $\mu$ in $\mathbb{G}_{N_{1}, \ldots, N_{k-1} ; i, p}$. By definition, we see that $\mu_{p}^{(1)}$ is of type $\mathrm{O}$ and $\mu_{j}^{(1)}$ is of type E, where $p+1 \leq j \leq N_{1}$. If $\mu_{p}^{(1)}$ is an overlined odd part, then set $\mu_{p}^{(1)}=\overline{2 t+1}$; If $\mu_{p}^{(1)}$ is a non-overlined even part, then set $\mu_{p}^{(1)}=2 t$, by definition of type $\mathrm{O}$, we see that there is an $s$-marked $\overline{2 t+1}$ in $\mu$, where $s \geq 2$.

For $1 \leq p \leq N_{1}$, define $\nu=\Theta_{p}(\mu)$ which can be obtained from $\mu$ as follows. There are three cases.

Case 1 If $1 \leq p<N_{1}$ and $\mu_{p}^{(1)}=\overline{2 t+1}$, then $\mu_{p+1}^{(1)}$ is of type E, set $\mu_{p+1}^{(1)}=2 b+2$, and it follows from the definition of Göllnitz-Gordon marking that $b \geq t+1$. There are two subcases.

Case 1.1 If $b=t+1$, that is, $\mu_{p+1}^{(1)}=2 t+4$, then replace $\mu_{p}^{(1)}=\overline{2 t+1}$ by $2 t+2$ and replace $\mu_{p+1}^{(1)}=2 t+4$ by $\overline{2 t+5}$.

Case 1.2 If $b>t+1$, and set $r$ to be the largest mark of the parts $2 b+2$ in $\mu$, then replace $\mu_{p}^{(1)}=\overline{2 t+1}$ by $2 t+2$ and replace the $r$-marked $2 b+2$ in $\mu$ by $\overline{2 b+3}$.

Case 2 If $1 \leq p<N_{1}$ and $\mu_{p}^{(1)}=2 t$, then $\mu_{p+1}^{(1)}$ is of type E, set $\mu_{p+1}^{(1)}=2 b+2$ and $b \geq t+1$. Note that there is an $s$-marked $\overline{2 t+1}$ in $\mu$. There are two subcases.

Case 2.1 If there is an $s$-marked $2 t+4$ in $\mu$, then replace the $s$-marked $\overline{2 t+1}$ in $\mu$ by $2 t+2$ and replace the $s$-marked $2 t+4$ in $\mu$ by $\overline{2 t+5}$.

Case 2.2 If there is no $s$-marked $2 t+4$ in $\mu$, and set $r$ to be the largest mark of the parts $2 b+2$ in $\mu$, then replace the $s$-marked $\overline{2 t+1}$ in $\mu$ by $2 t+2$ and replace the $r$-marked $2 b+2$ in $\mu$ by $\overline{2 b+3}$.

Case 3 If $p=N_{1}$, then there are two subcases.

Case 3.1 If $\mu_{p}^{(1)}=\overline{2 t+1}$, then replace $\mu_{p}^{(1)}=\overline{2 t+1}$ by $2 t+2$.

Case 3.2 If $\mu_{p}^{(1)}=2 t$, and there is an $s$-marked $\overline{2 t+1}$ in $\mu$, then replace the $s$-marked $\overline{2 t+1}$ in $\mu$ by $2 t+2$.

Obviously, when $1 \leq p<N_{1},|\nu|=|\mu|+2$, and when $p=N_{1},|\nu|=|\mu|+1$. We next show that the parts from $\mu$ in $\nu$ have the same marks as in $\mu$ and the new generated parts in $\nu$ replacing the parts in $\mu$ have the same marks as their original parts in $\mu$. This implies that $\nu \in \mathbb{G}_{N_{1}, \ldots, N_{k-1} ; i}$. By the definition of $\nu$, it is obvious that the marks of parts of size not exceed $2 t-1$ in $\nu$ stay the same as in $\mu$. We now consider the marks of the new generated parts in $\nu$. There are two cases:

- If $\mu_{p}^{(1)}=\overline{2 t+1}$, then $\left|\mu_{p-1}^{(1)}\right| \leq 2 t-1$, and so there is no 1 -marked $2 t$ in $\nu$. Hence the new generated part $2 t+2$ replacing $\mu_{p}^{(1)}=\overline{2 t+1}$ in $\mu$ should be marked with 1 
in $\nu$ and the parts $2 t+2$ from $\mu$ in $\nu$ have the same marks as in $\mu$. Thus, the marks of parts $2 t+2$ in $\nu$ stay the same as in $\mu$ when $\mu_{p}^{(1)}=\overline{2 t+1}$ for $1 \leq p \leq N_{1}$.

For Case 1.1, since $\nu_{p}^{(1)}=2 t+2$, it follows that the new generated part $\overline{2 t+5}$ replacing $\mu_{p+1}^{(1)}=2 t+4$ is marked with 1 in $\nu$.

For Case 1.2 , since $\nu_{p}^{(1)}=2 t+2$ and $\mu_{p+1}^{(1)}=2 b+2$, where $b>t+1$, it follows from the definition of Göllnitz-Gordon marking that the new generated part $\overline{2 b+3}$ replacing the $r$-marked $2 b+2$ in $\mu$ is marked with $r$ in $\nu$.

- If $\mu_{p}^{(1)}=2 t$, then there is an $s$-marked $\overline{2 t+1}$ in $\mu$. It follows from the definition of Göllnitz-Gordon marking that there are $f$-marked 2t's in $\mu$, where $1 \leq f \leq s-1$, and hence the new generated part $2 t+2$ replacing the $s$-marked $\overline{2 t+1}$ in $\mu$ is also marked with $s$ in $\nu$ and the marks of the parts $2 t+2$ from $\mu$ in $\nu$ are the same as in $\mu$ when $\mu_{p}^{(1)}=2 t$ for $1 \leq p \leq N_{1}$.

For Case 2.1, note that there is an $s$-marked $2 t+4$ in $\mu$, so there are $f$-marked $(2 t+4)$ 's in $\mu$, where $1 \leq f \leq s$, and the parts $2 t+4$ from $\mu$ in $\nu$ have the same marks as in $\mu$. Furthermore the new generated part $\overline{2 t+5}$ replacing the $s$-marked $2 t+4$ in $\mu$ is marked with $s$ in $\nu$.

For Case 2.2 , note that there are $f$-marked $(2 b+2)$ 's in $\mu$, where $1 \leq f \leq r$, so we derive that the parts $2 b+2$ from $\mu$ in $\nu$ have the same marks as in $\mu$ and the new generated part $\overline{2 b+3}$ in $\nu$ replacing the $r$-marked $2 b+2$ in $\mu$ should be marked with $r$ in $\nu$.

In any cases, we see that the marks of new generated parts in $\nu$ are the same as the marks of their original parts in $\mu$. Furthermore, the marks of the other parts from $\mu$ in $\nu$ are the same as in $\mu$. Hence $\nu \in \mathbb{G}_{N_{1}, \ldots, N_{k-1} ; i}$.

From the definition of $\nu$, it is easy to check that $\nu_{p}^{(1)}$ is of type E, $\nu_{p+1}^{(1)}$ is of type $\mathrm{O}$, and $\nu_{j}^{(1)}$ is of type $\mathrm{E}$, where $p+2 \leq j \leq N_{1}$. Hence, we deduce that $\nu \in \overline{\mathbb{G}}_{N_{1}, \ldots, N_{k-1} ; i, p}$. Therefore, $\Theta_{p}$ is well-defined. Furthermore, $\mu_{j}^{(1)}$ and $\nu_{j}^{(1)}$ are of the same type for $j \neq$ $p, p+1$.

To prove that $\Theta_{p}$ is a bijection, we give a brief description of the inverse map $\Lambda_{p}$ of $\Theta_{p}$ for $1 \leq p \leq N_{1}$. Let $\nu^{(1)}=\left(\nu_{1}^{(1)}, \nu_{2}^{(1)}, \ldots, \nu_{N_{1}}^{(1)}\right)$ be the first sub-overpartition of $\nu$ in $\overline{\mathbb{G}}_{N_{1}, \ldots, N_{k-1} ; i, p}$. By definition, $\nu_{p}^{(1)}$ is of type E, $\nu_{p+1}^{(1)}$ is of type $\mathrm{O}$, and $\nu_{j}^{(1)}$ is of type $\mathrm{E}$, where $p+2 \leq j \leq N_{1}$. For $1 \leq p<N_{1}$, if $\nu_{p+1}^{(1)}$ is an overlined odd part, then set $\nu_{p+1}^{(1)}=\overline{2 b+3}$; if $\nu_{p+1}^{(1)}$ is a non-overlined even part, then set $\nu_{p+1}^{(1)}=2 b+2$, by the definition of type $\mathrm{O}$, we see that there is an $r^{\prime}$-marked $\overline{2 b+3}$ in $\nu$, where $r^{\prime} \geq 2$.

For $1 \leq p \leq N_{1}$, define $\mu=\Lambda_{p}(\nu)$ which can be obtained from $\nu$ as follows. Here we set $\nu_{p}^{(1)}=2 t+2$. There are three cases. 
Case 1 If $1 \leq p<N_{1}$ and $\nu_{p+1}^{(1)}=\overline{2 b+3}$, then by the definition of Göllnitz-Gordon marking, we see that $t \leq b-1$. There are three subcases.

Case 1.1 If $t=b-1$, that is, $\nu_{p}^{(1)}=2 b$, then replace $\nu_{p+1}^{(1)}=\overline{2 b+3}$ by $2 b+2$ and replace $\nu_{p}^{(1)}=2 b$ by $\overline{2 b-1}$.

Case 1.2 If $t<b-1$ and $2 t+4$ does not occur in $\nu$, then replace $\nu_{p}^{(1)}=2 t+2$ by $\overline{2 t+1}$ and replace $\nu_{p+1}^{(1)}=\overline{2 b+3}$ by $2 b+2$.

Case 1.3 If $t<b-1$ and $2 t+4$ occurs in $\nu$, set $s^{\prime}$ to be the smallest mark of the parts $2 t+4$ in $\nu$, then replace $\nu_{p+1}^{(1)}=\overline{2 b+3}$ by $2 b+2$ and replace the $s^{\prime}$-marked $2 t+4$ in $\nu$ by $\overline{2 t+3}$.

Case 2 If $1 \leq p<N_{1}$ and $\nu_{p+1}^{(1)}=2 b+2$, then it follows from the definition of GöllnitzGordon marking that $t<b-1$ and there is an $r^{\prime}$-marked $\overline{2 b+3}$ in $\nu$. There are two subcases.

Case 2.1 If $2 t+4$ does not occur in $\nu$, then replace the $r^{\prime}$-marked $\overline{2 b+3}$ in $\nu$ by $2 b+2$ and replace $\nu_{p}^{(1)}=2 t+2$ by $\overline{2 t+1}$.

Case 2.2 If $2 t+4$ occurs in $\nu$ and set $s^{\prime}$ to be the smallest mark of the parts $2 t+4$ in $\nu$, then replace the $r^{\prime}$-marked $\overline{2 b+3}$ in $\nu$ by $2 b+2$ and replace the $s^{\prime}$-marked $2 t+4$ in $\nu$ by $\overline{2 t+3}$.

Case 3 If $p=N_{1}$, then there are two subcases.

Case 3.1 If $2 t+4$ does not occur in $\nu$, then replace $\nu_{p}^{(1)}=2 t+2$ by $\overline{2 t+1}$.

Case 3.2 If $2 t+4$ occurs in $\nu$, and set $s^{\prime}$ to be the smallest mark of the parts $2 t+4$ in $\nu$, then replace the $s^{\prime}$-marked $2 t+4$ in $\nu$ by $\overline{2 t+3}$.

It can be verified that $\Lambda_{p}(\nu) \in \mathbb{G}_{N_{1}, \ldots, N_{k-1} ; i, p}$ and $\Lambda_{p}$ is the inverse map of $\Theta_{p}$. So, we conclude that $\Theta_{p}$ is a bijection.

We conclude this section by presenting the following three examples for the bijection $\Theta_{p}$ in Lemma 5.2 .

(1) For $p=1$, let

$$
G G(\mu)=\left[\begin{array}{lllllll} 
& & & 8 & & & \\
& 2 & & 8 & & 12 & \\
\overline{\mathbf{1}} & & \mathbf{6} & & 10 & & 14
\end{array}\right] \begin{aligned}
& 3 \\
& 2 \\
& 1
\end{aligned}
$$

be the Göllnitz-Gordon marking representation of $\mu$ in $\mathbb{G}_{4,3,1 ; 3,1}$. Note that $\mu_{1}^{(1)}=\overline{1}$ and $\left|\mu_{2}^{(1)}\right|=6>4$, which satisfy the conditions in Case 1.2 in the definition of $\Theta_{p}$, so $r=1$. Applying the bijection $\Theta_{1}$ to $\mu$, we get

$$
G G(\nu)=\left[\begin{array}{llllll}
8 & & & & \\
2 & & 8 & & 12 & \\
2 & \overline{7} & & 10 & & 14
\end{array}\right] \begin{aligned}
& 3 \\
& 2 \\
& 1
\end{aligned},
$$


which is in $\overline{\mathbb{G}}_{4,3,1 ; 3,1}$. Note that $\nu_{1}^{(1)}=2$ and $\nu_{2}^{(1)}=\overline{7}$ and 4 does not occur in $\nu$, which satisfy the conditions in Case 1.2 in the definition of $\Lambda_{p}$. Applying $\Lambda_{1}$ to $\nu$, we recover $\mu$.

(2) For $p=3$, let

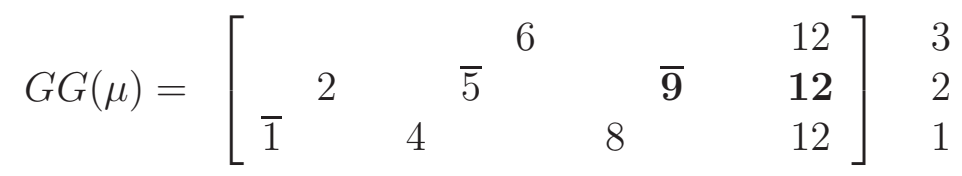

be the Göllnitz-Gordon marking representation of $\mu$ in $\mathbb{G}_{4,4,2 ; 3,3}$. Note that $\mu_{3}^{(1)}=8$, $\mu_{3}^{(2)}=\overline{9}$ and $\mu_{4}^{(2)}=12$, which satisfy the conditions in Case 2.1 in the definition of $\Theta_{p}$, so $s=2$. Employing the bijection $\Theta_{3}$ to $\mu$, we get

$$
G G(\nu)=\left[\begin{array}{lllllllll} 
& & & & & & & 12 & \\
& 2 & & \overline{5} & & & \mathbf{1 0} & & \overline{\mathbf{1 3}} \\
\overline{1} & & 4 & & & 8 & & 12 &
\end{array}\right] \begin{aligned}
& 3 \\
& 2 \\
& 1
\end{aligned},
$$

which is in $\overline{\mathbb{G}}_{4,4,2 ; 3,3}$. Note that $\nu_{3}^{(1)}=8, \nu_{3}^{(2)}=10, \nu_{4}^{(1)}=12$ and $\nu_{4}^{(2)}=\overline{13}$, which satisfy the conditions in Case 2.2 in the definition of $\Lambda_{p}$, so $r^{\prime}=2$. Applying $\Lambda_{3}$ to $\nu$, we recover $\mu$.

(3) For $p=4$, let

$$
G G(\mu)=\left[\begin{array}{llllllll} 
& \multicolumn{3}{c}{6} & & & & 12 \\
& 2 & & 6 & & & \overline{\mathbf{1 1}} & 3 \\
& & & & \overline{7} & 10 & &
\end{array}\right]
$$

be the Göllnitz-Gordon marking representation of $\mu$ in $\mathbb{G}_{4,3,2 ; 3,4}$. Note that $\mu_{4}^{(1)}=10$ and $\mu_{3}^{(2)}=\overline{11}$, which satisfy the conditions in Case 3.2 in the definition of $\Theta_{p}$, so $s=2$. Applying the bijection $\Theta_{4}$ to $\mu$, we get

$$
G G(\nu)=\left[\begin{array}{lllllll} 
& & & 6 & & & 12 \\
& 2 & & 6 & & & \mathbf{1 2} \\
\overline{1} & & 4 & & \overline{7} & 10 &
\end{array}\right] \begin{aligned}
& 3 \\
& 2 \\
& 1
\end{aligned},
$$

which is in $\overline{\mathbb{G}}_{4,3,2 ; 3,4}$. Note that $\nu_{4}^{(1)}=10$ and $\nu_{3}^{(2)}=12$, which satisfy the conditions in Case 3.2 in the definition $\Lambda_{p}$, so $s^{\prime}=2$. Applying $\Lambda_{4}$ to $\nu$, we recover $\mu$.

\section{Proof of Theorem 1.9}

In this section, we complete the proof of Theorem 1.9. Using Lemma 3.5 and Lemma 3.6, we first give a proof of the formula for the generating function of $F_{k, i}(m, n)$ in Theorem 3.4 . 
Proof of Theorem 3.4. First, we derive the following formula for the generating function of the number of overpartitions $\lambda$ in $\mathbb{E}_{N_{1}, \ldots, N_{k-1} ; i}$ with the aid of the identity (3.1) due to Kurşungöz.

$$
\sum_{\lambda \in \mathbb{E}_{N_{1}, \ldots, N_{k-1} ; i}} q^{|\lambda|}=\frac{q^{2\left(N_{1}^{2}+\cdots+N_{k-1}^{2}+N_{i}+\cdots+N_{k-1}\right)}}{\left(q^{2} ; q^{2}\right)_{N_{1}-N_{2}} \cdots\left(q^{2} ; q^{2}\right)_{N_{k-2}-N_{k-1}}\left(q^{2} ; q^{2}\right)_{N_{k-1}}}
$$

Recall that $\mathbb{B}_{N_{1}, \ldots, N_{k-1} ; i}$ is the set of ordinary partitions $\eta$ for which

$$
f_{1}(\eta) \leq i-1 \quad \text { and } \quad f_{t}(\eta)+f_{t+1}(\eta) \leq k-1
$$

such that there are $N_{r} r$-marked parts in the Gordon marking of $\eta$ for $1 \leq r \leq k-1$. To prove Theorem 1.4 combinatorially, Kurşungöz [18] established the following formula for the generating function of the number of ordinary partitions in $\mathbb{B}_{N_{1}, \ldots, N_{k-1} ; i}$.

$$
\sum_{\eta \in \mathbb{B}_{N_{1}, \ldots, N_{k-1} ; i}} q^{|\eta|}=\frac{q^{N_{1}^{2}+\cdots+N_{k-1}^{2}+N_{i}+\cdots+N_{k-1}}}{(q ; q)_{N_{1}-N_{2}} \cdots(q ; q)_{N_{k-2}-N_{k-1}}(q ; q)_{N_{k-1}}} .
$$

From the definitions in Section 3, we see that $\mathbb{E}_{N_{1}, \ldots, N_{k-1} ; i}$ is also the set of ordinary partitions $\lambda$ without odd parts for which

$$
f_{2}(\lambda) \leq i-1 \quad \text { and } \quad f_{2 t}(\lambda)+f_{2 t+2}(\lambda) \leq k-1
$$

such that there are $N_{r} r$-marked parts in the Göllnitz-Gordon marking of $\lambda$ for $1 \leq r \leq$ $k-1$.

To show (6.1), we aim to build a bijection $\phi$ between $\mathbb{E}_{N_{1}, \ldots, N_{k-1} ; i}$ and $\mathbb{B}_{N_{1}, \ldots, N_{k-1} ; i}$ such that for $\lambda \in \mathbb{E}_{N_{1}, \ldots, N_{k-1} ; i}$ and $\eta=\phi(\lambda) \in \mathbb{B}_{N_{1}, \ldots, N_{k-1} ; i}$, we have $|\lambda|=2|\eta|$.

In terms of generating functions, we have

$$
\sum_{\lambda \in \mathbb{E}_{N_{1}, \ldots, N_{k-1} ; i}} q^{|\lambda|}=\sum_{\eta \in \mathbb{B}_{N_{1}, \ldots, N_{k-1} ; i}} q^{2|\eta|} .
$$

Let $\lambda=\left(\lambda_{1}, \lambda_{2}, \ldots, \lambda_{\ell}\right)$ be a partition in $\mathbb{E}_{N_{1}, \ldots, N_{k-1} ; i}$, where $\lambda_{1} \leq \lambda_{2} \leq \cdots \leq \lambda_{\ell}$, we see that $\lambda_{j}$ is a non-overlined even part for $1 \leq j \leq \ell$ and $\lambda$ satisfies (6.4). Define

$$
\eta=\phi(\lambda)=\left(\frac{\lambda_{1}}{2}, \frac{\lambda_{2}}{2}, \ldots, \frac{\lambda_{\ell}}{2}\right) .
$$

Clearly, $|\lambda|=2|\eta|$. Furthermore, $f_{t}(\eta)=f_{2 t}(\lambda)$ for $t \geq 1$, which implies that $\eta$ satisfies (6.2). Hence it remains to show that the there are $N_{r} r$-marked parts in the Gordon marking of $\eta$ for $1 \leq r \leq k-1$.

By the definition of Göllnitz-Gordon marking, we see that the Göllnitz-Gordon marking of $\lambda=\left(\lambda_{1}, \lambda_{2}, \ldots, \lambda_{\ell}\right)$, where $\lambda_{j}$ is a non-overlined even part for $1 \leq j \leq \ell$ can be 
described as follows: First, $\lambda_{1}$ is marked with 1 , and for $p>1$, assume that the part $\lambda_{j}$ for $j<p$ has been assigned a mark. Then $\lambda_{p}$ is marked with the least positive integer that is not used to mark the parts $\lambda_{j}$ with $\lambda_{p}-\lambda_{j} \leq 2$ for $j<p$. For example, the Göllnitz-Gordon marking of $\lambda=(2,2,4,4,4,6,8,10,10,12,12,12)$ is

$$
G G(\lambda)=\left(2_{1}, 2_{2}, 4_{3}, 4_{4}, 4_{5}, 6_{1}, 8_{2}, 10_{1}, 10_{3}, 12_{2}, 12_{4}, 12_{5}\right) .
$$

We now consider the Gordon marking of $\eta=\left(\eta_{1}, \eta_{2}, \ldots, \eta_{\ell}\right)$ where $\eta_{1} \leq \eta_{2} \leq \cdots \leq \eta_{\ell}$. By definition, we see that $\eta_{1}$ is marked with 1 , and for $p>1$, assume that the part $\eta_{j}$ has been assigned a mark for $j<p$. Then $\eta_{p}$ is marked with the least positive integer that is not used to mark the parts $\eta_{j}$ with $\eta_{p}-\eta_{j} \leq 1$ for $j<p$. Since $\eta_{j}=\lambda_{j} / 2$ for $1 \leq j \leq \ell$, it can be checked that the mark of $\eta_{j}$ in the Gordon marking of $\eta$ is the same as the mark of $\lambda_{j}$ in the Göllnitz-Gordon marking of $\lambda$ for $1 \leq j \leq \ell$. For example, the Gordon marking of $\eta=\phi(\lambda)=(1,1,2,2,2,3,4,5,5,6,6,6)$ is

$$
G(\eta)=\left(1_{1}, 1_{2}, 2_{3}, 2_{4}, 2_{5}, 3_{1}, 4_{2}, 5_{1}, 5_{3}, 6_{2}, 6_{4}, 6_{5}\right)
$$

Hence there are $N_{r} r$-marked parts in the Gordon marking of $\eta$ for $1 \leq r \leq k-1$, and so $\eta \in \mathbb{B}_{N_{1}, \ldots, N_{k-1} ; i}$. Furthermore, it is easy to see that this process is reversible. Therefore, we conclude that $\phi$ is a bijection between $\mathbb{E}_{N_{1}, \ldots, N_{k-1} ; i}$ and $\mathbb{B}_{N_{1}, \ldots, N_{k-1} ; i}$, and (6.5) holds. Substituting (6.3) into (6.5), we obtain (6.1).

Substituting (6.1) into the relation (3.13) in Lemma 3.6, we obtain the following generating function of the number of overpartitions in $\mathbb{G}_{N_{1}, \ldots, N_{k-1} ; i}$.

$$
\sum_{\mu \in \mathbb{G}_{N_{1}, \ldots, N_{k-1} ; i}} q^{|\mu|}=\frac{\left(-q^{1-2 N_{1}} ; q^{2}\right)_{N_{1}} q^{2\left(N_{1}^{2}+\cdots+N_{k-1}^{2}+N_{i}+\cdots+N_{k-1}\right)}}{\left(q^{2} ; q^{2}\right)_{N_{1}-N_{2}} \cdots\left(q^{2} ; q^{2}\right)_{N_{k-2}-N_{k-1}}\left(q^{2} ; q^{2}\right)_{N_{k-1}}} .
$$

Plugging (6.6) into the relation (3.12) in Lemma 3.5, we obtain (3.11), which yields the generating function of $F_{k, i}(m, n)$ in Theorem 3.4. Thus we complete the proof.

By Theorem 3.4 and Lemma 3.3, we obtain the following generating function of $H_{k, i}(m, n)$.

Theorem 6.1. For $k \geq i \geq 1$,

$$
\begin{aligned}
& \sum_{m, n \geq 0} H_{k, i}(m, n) x^{m} q^{n} \\
& =\sum_{N_{1} \geq \cdots \geq N_{k-1} \geq 0} \frac{\left(-q^{2-2 N_{1}} ; q^{2}\right)_{N_{1}-1}\left(-q^{1-2 N_{1}} ; q^{2}\right)_{N_{1}} q^{2\left(N_{1}^{2}+\cdots+N_{k-1}^{2}+N_{i+1}+\cdots+N_{k-1}\right)} x^{N_{1}+\cdots+N_{k-1}}}{\left(q^{2} ; q^{2}\right)_{N_{1}-N_{2}} \cdots\left(q^{2} ; q^{2}\right)_{N_{k-2}-N_{k-1}}\left(q^{2} ; q^{2}\right)_{N_{k-1}}} .
\end{aligned}
$$


Proof. From the relation (3.7) in Lemma 3.3, we deduce that for $1 \leq i \leq k-1$,

$$
\begin{aligned}
& \sum_{m, n \geq 0} H_{k, i}(m, n) x^{m} q^{n} \\
& =\sum_{m, n \geq 0} F_{k, i+1}(m, n) x^{m} q^{n} \\
& =\sum_{N_{1} \geq \cdots \geq N_{k-1} \geq 0} \frac{\left(-q^{2-2 N_{1}} ; q^{2}\right)_{N_{1}-1}\left(-q^{1-2 N_{1}} ; q^{2}\right)_{N_{1}} q^{2\left(N_{1}^{2}+\cdots+N_{k-1}^{2}+N_{i+1}+\cdots+N_{k-1}\right)} x^{N_{1}+\cdots+N_{k-1}}}{\left(q^{2} ; q^{2}\right)_{N_{1}-N_{2}} \cdots\left(q^{2} ; q^{2}\right)_{N_{k-2}-N_{k-1}}\left(q^{2} ; q^{2}\right)_{N_{k-1}}} .
\end{aligned}
$$

For $i=k$, from (3.8) in Lemma 3.3, it follows that

$$
\sum_{m, n \geq 0} H_{k, k}(m, n) x^{m} q^{n}=\sum_{m, n \geq 0} F_{k, 1}(m, n)\left(x q^{-2}\right)^{m} q^{n}
$$

Using the generating function of $F_{k, 1}(m, n)$, we obtain

$$
\begin{aligned}
& \sum_{m, n \geq 0} H_{k, k}(m, n) x^{m} q^{n} \\
& =\sum_{N_{1} \geq \cdots \geq N_{k-1} \geq 0} \frac{\left(-q^{2-2 N_{1}} ; q^{2}\right)_{N_{1}-1}\left(-q^{1-2 N_{1}} ; q^{2}\right)_{N_{1}} q^{2\left(N_{1}^{2}+\cdots+N_{k-1}^{2}\right)} x^{N_{1}+\cdots+N_{k-1}}}{\left(q^{2} ; q^{2}\right)_{N_{1}-N_{2}} \cdots\left(q^{2} ; q^{2}\right)_{N_{k-2}-N_{k-1}}\left(q^{2} ; q^{2}\right)_{N_{k-1}}} .
\end{aligned}
$$

Observe that the above formula (6.8) for $1 \leq i \leq k-1$ and (6.9) for $i=k$ take the same form as in Theorem 6.1. Thus, we complete the proof of Theorem 6.1 .

We conclude this paper with the proof of Theorem 1.9.

Proof of Theorem 1.9. Substituting (3.9) and (6.7) into the relation (3.6), we obtain

$$
\begin{aligned}
& \sum_{m, n \geq 0} O_{k, i}(m, n) x^{m} q^{n} \\
& =\sum_{m, n \geq 0} F_{k, i}(m, n) x^{m} q^{n}+\sum_{m, n \geq 0} H_{k, i}(m, n) x^{m} q^{n} \\
& =\sum_{N_{1} \geq \cdots \geq N_{k-1} \geq 0} \frac{\left(-q^{2-2 N_{1}} ; q^{2}\right)_{N_{1}-1}\left(-q^{1-2 N_{1}} ; q^{2}\right)_{N_{1}} q^{2\left(N_{1}^{2}+\cdots+N_{k-1}^{2}+N_{i+1}+\cdots+N_{k-1}\right)}\left(1+q^{2 N_{i}}\right) x^{N_{1}+\cdots+N_{k-1}}}{\left(q^{2} ; q^{2}\right)_{N_{1}-N_{2}} \cdots\left(q^{2} ; q^{2}\right)_{N_{k-2}-N_{k-1}}\left(q^{2} ; q^{2}\right)_{N_{k-1}}},
\end{aligned}
$$

which is (1.6). This completes the proof.

Acknowledgments. This work was supported by the 973 Project, the PCSIRT Project and the National Science Foundation of China. We are greatly indebted to referees for their helpful suggestions that improved the presentation of this paper. 


\section{References}

[1] A.K. Agarwal, G.E. Andrews and D.M. Bressoud, The Bailey lattice, J. Ind. Math. Soc. 51 (1987) 57-73.

[2] G.E. Andrews, An analytical proof of the Rogers-Ramanujan-Gordon identities, Amer. J. Math. 88 (1966) 844-846.

[3] G.E. Andrews, A generalization of the Göllnitz-Gordon partition theorem, Proc. Amer. Math. Soc. 18 (1967) 945-952.

[4] G.E. Andrews, An analytic generalization of the Rogers-Ramanujan identities for odd moduli, Proc. Nat. Acad. Sci. USA 71 (1974) 4082-4085.

[5] G.E. Andrews, The Theory of partitions, Addison-Wesley Publishing Co., 1976.

[6] G.E. Andrews, Multiple series Rogers-Ramanujan type identities, Pacific J. Math. 114 (1984) 267-283.

[7] G.E. Andrews, q-Series: Their Development and Application in Analysis, Number Theory, Combinatorics, Physics, and Computer Algebra, American Mathematical Soc, 1986.

[8] G.E. Andrews, Bailey's transform, lemma, chains and tree, In: Special Functions 2000, Kluwer Acad. Publ., Dordrecht, 2001, pp. 1-22.

[9] W.N. Bailey, Identities of the Rogers-Ramanujan type, Proc. London Math. Soc. 50(2) (1949) 1-10.

[10] D.M. Bressoud, Analytic and combinatorial generalizations of Rogers-Ramanujan identities, Mem. Amer. Math. Soc. 24(227) (1980) 54pp.

[11] D.M. Bressoud, M. Ismail and D. Stanton, Change of base in Bailey pairs, Ramanujan J. 4 (2000) 435-453.

[12] W.Y.C. Chen, D.D.M. Sang and D.Y.H. Shi, The Rogers-Ramanujan-Gordon thoerem for overpartitions, Proc. London Math. Soc. 106 (3) (2013) 1371-1393.

[13] S. Corteel and O. Mallet, Overpartitions, lattice paths and Rogers-Ramanujan identities, J. Combin. Theory Ser. A 114 (8) (2007) 1407-1437.

[14] S. Corteel, J. Lovejoy, and O. Mallet, An extension to overpartitions of the RogersRamanujan identities for even moduli, J. Number Theory 128 (2008) 1602-1621.

[15] H. Göllnitz, Partitionen mit differenzenbedingungen, J. Reine Angew. Math. 225 (1967) 154-190.

[16] B. Gordon, A combinatorial generalization of the Rogers-Ramanujan identities, Amer. J. Math. 83 (1961) 393-399.

[17] B. Gordon, Some continued fractions of the Rogers-Ramanujan type, Duke Math. J. 31 (1965) 741-748.

[18] K. Kurşungöz, Parity considerations in Andrews-Gordon identities, European J. Combin. 31 (2010) 976-1000.

[19] J. Lovejoy, Gordon's theorem for overpartitions, J. Combin. Theory, Ser. A 103 (2003) 393-401. 
[20] J. Lovejoy, Overpartition theorems of the Rogers-Ramanujan type, J. London Math. Soc. 69 (2004) 562-574.

[21] J. Lovejoy, A Bailey lattice, Proc. Amer. Math. Soc. 132 (2004) 1507-1516.

[22] J. Lovejoy, Partitions and overpartitions with attached parts, Arch. Math. (Basel) 88 (2007) 316-322.

[23] J. Lovejoy, Partitions with rounded occurrences and attached parts, Ramanujan J. 23 (2010) 307-313.

[24] P. Paule, A note on Bailey's lemma, J. Combin. Theory Ser. A 44 (1987) 164-167.

[25] L.J. Slater, Further identities of Rogers-Ramanujan type, Proc. London Math. Soc. 54(2) (1952) 147-167.

[26] S.O. Warnaar, 50 years of Bailey's lemma, In: Algebraic combinatorics and applications, Springer, Berlin, 2001, pp. 333-347. 